\title{
A combined computational and experimental characterization of lean premixed turbulent low swirl laboratory flames II. Hydrogen flames
}

\author{
Marc Day ${ }^{*}$, Shigeru Tachibana ${ }^{\dagger}$, John Bell ${ }^{*}$, Michael Lijewski, Vince Beckner ${ }^{*}$, and Robert K. Cheng ${ }^{\ddagger}$ \\ ${ }^{*}$ Center for Computational Science and Engineering, Lawrence Berkeley National Laboratory, Berkeley, CA, 94720 \\ ${ }^{\dagger}$ Institute of Aeronautical Technology, Japan Aerospace Exploration Agency, 7-44-1 Jindaiji-Higashii, Chofu, Tokyo \\ 182-8522, Japan \\ ${ }^{\ddagger}$ Environmental Energy Technologies Div., Lawrence Berkeley National Laboratory, Berkeley, CA, 94720
}

\begin{abstract}
We present simulations of laboratory-scale Low Swirl Burner (LSB) flames in order to develop a characterization of the interaction of thermal/diffusive unstable flames with turbulence at the correct scales of laboratory experiments. A Lagrangian diagnostic was developed to overcome the pitfalls of traditional Eulerian analysis techniques when applied to cellular flame systems, including the lack of a well-defined measure of "flame progress" and the timedependent strain and curvature fields that evolve at scales that are faster than the residence time in the flame zone. An integrated measure of consumption along the pathlines was shown to serve as a generalized analog of the Euleriancomputed consumption-based burning speed. The diffusive fuel flux divergence along the pathlines was shown to correlate directly with integrated consumption rate. Insights gained through the Lagrangian diagnostic analysis served as the underpinning of a new procedure to interpret OH-PLIF images from the LSB experiment. This new diagnostic is able to provide a more physically meaningful approximation to the "flame surface area" than traditional approaches based on PIV processing.
\end{abstract}

\section{Introduction}

This paper is the second in a series that presents a combined computational and experimental study to investigate and characterize the structure of premixed turbulent $\mathrm{CH}_{4} / \mathrm{H}_{2}$ flames. The complete study consists of lean $\mathrm{CH}_{4}$-air flames that are thermal/diffusive neutral (Lewis number, $\mathrm{Le} \approx 1$ ), lean $\mathrm{H}_{2}$-air flames $(\mathrm{Le}<1$ ) that are thermal/diffusive unstable, and several flames of $\mathrm{CH}_{4}-\mathrm{H}_{2}$-air with stability properties intermediate to those of the two extremes. In our first paper, focused on $\mathrm{CH}_{4}$-air flames [1], we introduced the computational approach used to simulate the entire class of relevant laboratory-scale flames, and then discussed a number of diagnostics that validate the simulations with data taken from laboratory experiments. In the present study, a separate treatment of turbulent flames burning in hydrogenair mixtures is presented. The analysis of the diagnostics for both numerical and experimental results is significantly more complex due to the thermal/diffusive instability of the flame. It will be shown that the numerical results are invaluable to the interpretation of the experimental data.

Our previous investigations of Lewis number effects in simplified and laboratory scale configurations provide the framework for the current analysis [2, 3, 4, 5]. In these studies we employ a low Mach number formulation of the time-dependent reacting flow equations, and use adaptive mesh refinement (AMR) to evolve the system numerically. 
By combining the low Mach number model with AMR, the simulations can cover a physical domain that is an order of magnitude larger in each dimension than typical reacting flow direct numerical simulations (DNS) to date using comparable resources, while at the same time enabling the structure of the flame to be resolved using detailed models for the chemical kinetics and molecular transport.

In Reference [6] a computational study presents the dependence of flame structure on fuel Lewis number in a quasisteady 2D rectangular domain. The resulting lean $\mathrm{CH}_{4}$ and $\mathrm{C}_{3} \mathrm{H}_{8}$ flames exhibited local heat release characteristics that were similar to those of the corresponding steady unstrained 1D idealized flames. However, lean $\mathrm{H}_{2}$ flames are thermodiffusively unstable, a property which leads to considerable increases in the local heat release rate over much of the flame, as well as significant variation in the local heat release and fuel consumption rates, even in regions where the flame is locally flat. The tendency for such low Lewis number flames to form these highly nonuniform burning patterns is well-documented (see for example, the early theoretical works by Zeldovich [7]; Markstein [8]). These flames have long since been observed experimentally (for example, see Bregeon et al. [9] and Mitani \& Williams [10]), and have been the subject of many computational investigations, notably including the early work of Baum et al. [11] in an idealized configuration, and proceeding through the line of the author's own works cited above, which were conducted both in idealized and realistic geometrical configurations.

One previous 3D computational study [3], explored the thermal/diffusive instability effects of lean $\mathrm{H}_{2}$-air flames in an idealized configuration over a range of low-level turbulence intensities. In this study, cellular patterns of fuel consumption and flame propagation were observed as they interacted with the evolving turbulent flow field. A local fuel-consumption-based flame propagation speed, $S_{c}$, was defined for the flame, and values of $S_{c}$ were shown to be considerably higher in localized pockets than in the corresponding idealized flat flame. The enhancement in $S_{c}$ was attributed to localized fuel enrichment that resulted from differential diffusion processes. Enhanced global fuel consumption rates were attributed to the combination of increased flame surface area and intensified local consumption. The main implication of that work, practically, is that estimates of the turbulent flame speed as the product of flame surface area and laminar flame speed significantly under-predict the overall heat release and fuel consumption rates in these mixtures.

The overall structure of laboratory scale $\mathrm{H}_{2}$-air flames generated by a low-swirl burner (LSB) was described in Ref. [4]. From a snapshot in time, the cross-sectional slice of the computed flame solution shows discontinuities in both the $\mathrm{OH}$ concentration and the destruction rate of $\mathrm{H}_{2}$ along the reaction front; these discontinuities bound so-called "flame cells" that are characteristic of low Lewis number flames. Considerable variations in local post-flame temperatures along the cellular flame structures are also evident. When compared to experimental results from planar laser induced fluorescence of $\mathrm{OH}$ radicals (OH-PLIF), the simulation is shown to have captured the discontinuous features of this flame with remarkably similar size, shape and global structure. Further analysis performed on an isotherm surface of $\mathrm{T}=1144 \mathrm{~K}$ shows the cellular sizes of the laboratory-scale flame are smaller than those observed in the idealized simulations at lower turbulence intensties (see Day, et al. [3]). $S_{c}$ computed by locally integrating fuel consumption rates normal to the reaction front shows a range of 2 to 4 times the laminar flame speed, $S_{l}$, of an idealized unstretched laminar 1D flame. The similarities between measurement and simulations of the variations in fuel consumption rate and $\mathrm{OH}$ concentration, $X_{O H}$, along the flame front was also examined. Joint probability distribution functions (JPDFs) showed strong correlations between local burning speed and peak values of $X_{O H}$, taken locally normal to the flame front. This study provides the foundation for our current study to investigate $\mathrm{H}_{2}$ flame structures by using pathline diagnostics of the 3D time dependent computed flame solutions of two flames at different inflow turbulence intensities.

As indicated above, the goal of the present series of studies involving simulations of laboratory-scale LSB flames $[12,13,14]$ is to understand and characterize turbulence/flame interactions at the correct physical scales of laboratory experiments. For premixed combustion systems, this is a key feature since burners with different flame stabilization methods emphasize distinct aspects of the interplay between the mean and fluctuating flow features and flame propagation. Historically, it has been very difficult to characterize the wide range of experimental configurations with flames in a generic (device-neutral) context. High-fidelity simulations capable of capturing the interplay between the turbulent flow and chemistry at realistic experimental device scales can play an important role in providing useful insights for the development of turbulence and flame models to improve the fidelity of computational design tools for engineering systems, and will be of great aid in constructing the device-neutral characterizations that are presently lacking. 
The LSB establishes a radially divergent turbulent flow of reactants that aerodynamically stabilizes a detached freely-propagating premixed flame, as shown in (Fig. 1c). To facilitate laser diagnostic access the LSB flames burn in the open. Therefore, the swirling component of the mean flow interacts with the ambient air in the outer shear region. However, the central non-swirling region of the LSB is essentially isolated from the outer flow, and is devoid of complex large scale flow structures, such as an intense recirculation zone or coherent bulk motions. Notably, the LSB flame does not require an additional energy source such as a pilot flame at very lean fueling conditions in order to maintain flame stability. Also, self-similarity of the mean flowfield enables the LSB device to operate over a wide range of fuel mixtures, flow velocities, equivalence ratios, device pressures and fuel temperatures. This robustness allows lean methane and hydrogen fuels to be studied at similar inflow and turbulent conditions [15].

Similar to the approach of our first paper on $\mathrm{CH}_{4}$ [1] we compare simulation results of $\mathrm{H}_{2}$ flames with velocity statistics that are measured in the laboratory using particle image velocimetry (PIV). Flame front topologies are measured using OH-PLIF. To extract 3D time dependent flame information from numerical simulations of these flames, we applied the Lagrangian pathline tracking approach introduced in [16] and applied to turbulent methane-air flames in [1]. As discussed in Ref. [1], this diagnostic provides a unique, fluid-centric view of the flame that is complimentary to an iso-surface approach. In the context of $\mathrm{H}_{2}$ flames, analyses of the time-dependent transport of species from the Lagrangian pathlines give rise to local parameters for characterizing the statistical variations in the burning rates in $\mathrm{H}_{2}$ flames caused by thermal/diffusive effects. The numerical results also aid in the interpretation and analysis of the experimental data obtained from OH-PLIF, as will be discussed below.

\section{Numerical simulation}

A cross setional view of the LSB for our studies is shown in Fig. 1a. Details are described Ref. [14, 1]. Its nozzle has an inner diameter of $50 \mathrm{~mm}$, with a swirler placed $68 \mathrm{~mm}$ upstream of the exit plane. The annular section of the swirler (Fig. 1b) is fitted with eight constant thickness curved vanes, each having a discharge angle of $37^{\circ}$. The central channel is $38 \mathrm{~mm}$ in diameter, and is fit with a perforated plate that has 37 holes of $3 \mathrm{~mm}$ diameter. The geometric swirl number of this LSB is 0.55 according to the measurements given in Ref. [14]. As discussed in Appendix A of Day et al. [1], the flowfield generated by this LSB assumes self-similarity when $U_{0}>10 \mathrm{~m} / \mathrm{s}$.

The numerical simulations for this study use the low Mach number adaptive mesh refinement code, LMC, documented in Ref. [17]. The LMC code integrates the multi-species Navier-Stokes equations with chemical reactions, treating the fluid as a mixture of perfect gases, and using a mixture-averaged model for differential species diffusion. A comprehensive model [18] was used to describe the hydrogen combustion kinetics. Soret and Dufour effects and radiative transport are ignored, as discussed in further detail below. The low Mach number approximation [19, 20] exploits the natural separation of scales between fluid motion and acoustic wave propagation, removing acoustic wave propagation from the analytic description of the system. Bulk compressibility effects due to chemical reaction and thermal conduction remain in the description but appear as a global constraint on the evolution of the velocity field. A predictor-corrector procedure is used that is based on a density-weighted projection scheme [21]. For the species conservation equations a spectral deferred correction approach [22] is used to couple the different temporal scales associated with detailed kinetics, transport and advection. Time evolution of the overall scheme is constrained by the fluid velocity rather than the speed of sound, increasing the allowable numerical time step by a factor of approximately 10-20 compared to that required for traditional simulation approaches based on the compressible flow equations.

The most general form for the diffusive flux includes Soret, Dufour and barodiffusion terms. The barodiffusion terms vanish in the low Mach number limit. A number of studies have shown Soret transport to play a significant role in steady 1D lean hydrogen-air flames (cf., [23]), and in 2D naturally-propagating ([24]) hydrogen-air flames. In 1D steady flames the Soret effect significantly impacts predicted extinction strain rates. In 2D, the effect results in significantly enhanced local temperature gradients and up to $20 \%$ increases in local burning speeds. However, it has also been shown [25] that these effects are filtered away when considering mean properties of a fluctuating flame, resulting in less than $7 \%$ corrections to predicted mean flame features. We ignore Soret effects for the present work, 
and arrive at a Fickian approximation for diffusive mass flux, $\Gamma_{m}^{*}$, corrected to conserve mass

$$
\Gamma_{m} \approx \Gamma_{m}^{*}+\Gamma_{m}^{c}=-\rho \mathcal{D}_{m} \nabla X_{m}+\Gamma_{m}^{c},
$$

where $\mathcal{D}_{m}$ is the mixture-averaged diffusion coefficient for species $m, \rho$ is the local fluid mass density, and $X_{m}$ is the species mole fraction. $\Gamma_{m}^{c}=Y_{m} \sum \Gamma_{m}^{*}$ is a correction that ensures $\sum \Gamma_{m}=0$ (see [26] for a detailed derivation and justification of this approximation.). The mixture-averaged diffusion coefficients are given as part of the comprehensive model for hydrogen combustion, [18].

The adaptive implementation of LMC is based on a block-structured adaptive mesh refinement (AMR) strategy. In this approach, regions to be refined are organized into rectangular patches, with several thousand grid points per patch. One is thus able to use well-characterized rectangular grid methods to advance the solution in time; furthermore, the overhead in managing the irregular data structures is amortized over relatively large amounts of floating-point work. Adaptive refinement is performed in time as well as in space; each level of refinement is advanced at its own time step, subject to the constraint that the time step at a coarse level be an integral multiple of the time step at the next finer level. A synchronization strategy ensures that discrete conservation and the low Mach number constraint are enforced across all refinement levels during the simulation. Grid refinement criteria based on the evolving solution are used to dynamically adjust the location of the fine grids as the computation proceeds. For this study, refinement was focused around the flame front and in the turbulent reactants stream between the nozzle and the flame. The use of AMR results in more than an order of magnitude additional savings in computational resources compared to a uniform fine grid calculation of the same effective resolution.

LMC runs on distributed-memory parallel architectures using a dynamic load balancing algorithm that accommodates the changing workload as regions of refinement are created and destroyed during the computation. Here the load-balancing problem is complicated by the heterogeneous workloads associated with chemical kinetics. The overall scaling behavior of the code, in conjunction with the efficiency gains resulting from the low Mach number formulation and the use of adaptive refinement makes this code particularly well-suited for this study.

\section{Computational Setup}

There are several approaches to treat the LSB nozzle in DNS simulations. One possibility is to model the flow generated in the nozzle as part of the simulation; an alternative is to use measured data to prescribe the flow at the nozzle exit. In a series of studies [27, 28, 29], Nogenmyr et al. considered both options in the context of LES simulations of lean $\mathrm{CH}_{4}$-air mixtures. Considerably better agreement was found between simulated and measured profiles when the flow generated inside the nozzle was included. In particular, the simulations correctly predicted large-scale structures emanating from the swirl vanes, and these structures ultimately played a key role in stabilizing their flame. More generally, these results suggest that if experimental data is being used to define input conditions for a detailed flow simulation then a detailed experimental characterization of the nozzle flow is necessary, in terms of both mean profiles and turbulent fluctuations.

For the present simulations we incorporate an experimental characterization of the flow at the nozzle exit similar to that used in the Nognmeyr studies. Stereoscopic-PIV is not available at LBNL, so the 3D PIV data obtained by Petersson et al. [14] were used: mean velocity profiles were specified as functions of radius from the device centerline based on a lean $(\phi=0.62) \mathrm{CH}_{4}$ flame at a mean fueling rate of $U_{0}=6.2 \mathrm{~m} / \mathrm{s}$. We note that the experimental data showed significant azimuthal variations; clear imprints of the 8 swirl vanes are easily discernable in PIV data taken in a horizontal plane near the nozzle exit. Profiles of mean velocity in the experiment were formed by averaging the experimental measurements taken on two vertical PIV imaging planes: one that aligned with the swirl vanes, and one that was midway between a pair of them. To generate the simulation inlet data at $U_{0}=10$ and $15 \mathrm{~m} / \mathrm{s}$, the averaged flow profiles were scaled linearly to match the mean fueling rate while preserving the effective swirl number. We note that this scaling provides only a rough approximation to the mean flow because the flow field is not fully developed unless $U_{0} \geq 10 \mathrm{~m} / \mathrm{s}$., as discussed in Appendix A of Day et al. [1]. Thus, the resulting computations here are subject to the same limitations as those detailed in the reference. Similar to the earlier study on methane-air flames, the approach 
taken here with hydrogen-air flames is to first validate that the simulations are properly capturing the global properties of the experimental flames, but then to focus the investigation on the statistics of the local flame structure, rather than on the detailed interactions with the turbulent flow field across all length and time scales.

Turbulent fluctuations in the nozzle were created for the simulation in an auxiliary calculation and were superimposed on the mean flow profiles in order to simulate the effect of the upstream turbulence-generation plates. To do this, a zero-mean velocity field was generated that was representative of flow through a perforated plate (i.e., an array of small jets having the diameter and separation of the perforations). The flow was evolved numerically in a triply-periodic domain until the measured integral length scale in the direction of the jets was $4 \mathrm{~mm}$, consistent with experimental measurements taken at the core region of the nozzle in inert flow. The resulting turbulent flow field was then superimposed on the experimental profiles of mean flow, assuming a Taylor hypothesis [30]. A radial scaling factor was used to shape the synthetic fluctuations to the mean measured turbulence intensity profiles.

The reacting flow simulation was performed in cubical domain with an edge length $25 \mathrm{~cm}$. We implicitly assume that the boundaries of this box are sufficiently far from the flame that the boundary has no effect on the dynamics. The exit of the LSB nozzle is centered on the base plane of the computational domain, and provides the time-dependent inflow of turbulent swirling flow. Outside the LSB nozzle, a $0.35 \mathrm{~m} / \mathrm{s}$ upward coflow of cold air is specified. Note that this coflow configuration is consistent with the Lund experiments which provided the mean inlet flow profiles, but not with the LBNL configuration. Numerically, the small coflow helps to economize the computation time required to flush the initial transient data from the simulation domain without adversely affecting the swirling flow and flame stabilization in the core region. The top and lateral boundaries are outflow in this configuration.

In the simulations, we consider two inflow velocities, $10 \mathrm{~m} / \mathrm{s}$ and $15 \mathrm{~m} / \mathrm{s}$, corresponding to cases $\mathrm{H}-\mathrm{C}$ and $\mathrm{H}$ $\mathrm{D}$ in Table 1 below (Note that the table includes two parameters, $a_{z}$ and $z_{0}$ that are discussed in section 5). For each case the effective resolution for the simulation is $2048^{3}$ with uniform cells measuring $122.1 \mu \mathrm{m}$ on a side. This was accomplished using a base (coarse) mesh of $256^{3}$ uniform cells to cover the entire computational domain, and three additional levels of factor-of-two grid refinement to dynamically track regions of high vorticity (turbulence) and chemical reactivity (flame zone). For reference, the thermal thickness of the $\phi=0.37 \mathrm{H}_{2}$-air flames simulated in this study (Table 1) is approximately 800 microns. Locally, these lean $\mathrm{H}_{2}$-air fuels form cellular flames structures that can be almost a factor of two thinner [31]. The AMR refinement strategy we used explicitly enforced that regions of nontrivial fuel consumption (i.e., the "flame zone") were contained entirely within the finest level at all times (i.e., the simulation automatically refines all cells where the local fuel consumption was more than $0.01 \%$ of the peak value in the domain.). At this grid resolution, the thermal and fuel profiles of these lean hydrogen-air flames, as well as the Kolmogorov scales in the highest turbulent case, are well-resolved, as demonstrated explictly in [32]. The simulations were run from an arbitrary initial condition until the flame became statistically stationary (as indicated by the total inventory of fuel in the computational domain). We then continued the simulations to collect flow and flame statistics. Case H-C consumed approximately $850 \mathrm{~K}$ node hours on the Franklin machine at the NERSC facility. Approximately $100 \mathrm{~ms}$ of physical time was evolved at an effective resolution of $1024^{3}$. An additional $5 \mathrm{~ms}$ was evolved at the finest level, generating approximately 6TB of data for detailed analysis. Case H-D was evolved over $64 \mathrm{~ms}$ at $1024^{3}$, and an additional $1 \mathrm{~ms}$ at $2048^{3}$. This case consumed approximately $1.5 \mathrm{M}$ node hours on Franklin and generated approximately $8 \mathrm{~TB}$ for analysis. Laboratory diagnostics were collected for case H-E, and are discussed below, but case H-E was not simulated.

Table 1: Conditions of the experimental and simulated $\phi=0.37 \mathrm{H}_{2}$-air flames. (Note: the quantites $a_{z}$ and $z_{0}$ are discussed in section 5)

\begin{tabular}{|c|c|c|c|c|c|c|c|c|}
\hline \multirow[b]{2}{*}{ Case } & \multirow[b]{2}{*}{$U_{0}(\mathrm{~m} / \mathrm{s})$} & \multicolumn{5}{|c|}{ Measured } & \multicolumn{2}{|l|}{ Simulated } \\
\hline & & $u_{z}^{\prime}(\mathrm{m} / \mathrm{s})$ & $u_{r}^{\prime}(\mathrm{m} / \mathrm{s})$ & $\mathrm{Re}$ & $a_{z}(1 / \mathrm{mm})$ & $z_{0}(\mathrm{~mm})$ & $a_{z}(1 / \mathrm{mm})$ & $z_{0}(\mathrm{~mm})$ \\
\hline $\mathrm{H}-\mathrm{C}$ & 10 & 0.715 & 0.511 & 119 & -0.0119 & -48.1 & -0.0134 & -32.46 \\
\hline H-D & 15 & 1.08 & 0.74 & 163 & -0.0112 & -53.0 & -0.0146 & -28.15 \\
\hline H-E & 18 & 1.16 & 0.87 & 221 & -0.0115 & -52.0 & & \\
\hline
\end{tabular}




\section{Diagnostics and experimental setup}

The scalar and velocity fields of the laboratory LSB flame experiments were measured using OH-PLIF and PIV. Details of the OH-PLIF and PIV setups can be found respectively in Cheng et al. [33] and Johnson and Cheng [34]. The OHPLIF system consists of a Spectra Physics pulsed Nd:Yag laser pumping a Quanta Ray dye laser with Rhodamine 590 dye. The output of the dye laser is frequency doubled and tuned to the $\mathrm{P}_{1}(2)$ absorption $(282.58 \mathrm{~nm})$ of the $\mathrm{OH}(1,0)$ band of the $A^{2} \Sigma^{+}-X^{2} \Pi$ system. The laser beam is shaped into a thin $(200 \mathrm{~nm})$ vertical sheet passing through the LSB axis. The $\mathrm{OH}$ fluorescence signal from the flame is detected by an intensified Xybion camera through band pass filters (Schott UG-5 and WG-305). The camera captures a field-of-view of $r=20 \mathrm{~mm}$ and $z=27 \mathrm{~mm}$ at a resolution of $60 \mathrm{~mm} /$ pixel. For each flame, 500 images are obtained for the analysis.

The PIV system uses a New-Wave Solo Nd:Yag laser with double $120 \mathrm{~mJ}$ pulses at $532 \mathrm{~nm}$ and a Kodak/Red Lake ES 4.0 digital camera with 2048 by 2048 pixel resolution. The optics was configured to capture a field of view of approximately $13 \mathrm{~cm}$ by $13 \mathrm{~cm}$ covering the nearfield as well as the farfield of the flames with $0.065 \mathrm{~mm} /$ pixel resolution. A cyclone-type particle seeder supplies the air flow with Aerosil amorphous silica particles. For each flame, 224 pairs of PIV images are obtained for velocity statistical analysis. As reported by Peterssen et al., [14], the asymmetry of swirler geometry imposes azimuthal variations in the velocity and the scalars fields. To be consistent with the prior studies, the OH-PLIF and PIV image planes were centered on the only pair of vanes that are aligned with a row of holes on the perforated plate.

In the experiment, three flames (H-C, $\mathrm{H}-\mathrm{D}$, and $\mathrm{H}-\mathrm{E}$ ) were investigated with a $\phi=0.37 \mathrm{H}_{2}$-air mixture at flow velocities, $U_{0}$, of 10,15 , and $18 \mathrm{~m} / \mathrm{s}$ (Table 1 ). Lower velocity cases (i.e. 3 and $6 \mathrm{~m} / \mathrm{s}$ corresponding to previous $\mathrm{CH}_{4}$ flames M-A and M-B, [1]) could not be performed because of $\mathrm{H}_{2}$ flame flashback when $U_{0}<10 \mathrm{~m} / \mathrm{s}$. As reported in Day et al. [1], the non-reacting flowfield only becomes fully developed at $U_{0}>10 \mathrm{~m} / \mathrm{s}$. Therefore, the simulations performed for two flames, H-C and H-D at $U_{0}=10$ and $15 \mathrm{~m} / \mathrm{s}$, are within the fully developed flow regime.

The turbulence parameters $u_{z}^{\prime}$, and $u_{r}^{\prime}$ in Table 1, measured on the centerline at the leading edges of the flame brushes according to the procedure of Ref. [12], are the appropriate representations of the initial conditions of these LSB flames. The integral length scale for the calculation of Re, was deduced from analysis PIV measurements taken for a non-reacting flow (Appendix 1 in Day, et al. [1]). The Damkholer number, Da, and Karlovitz number, Ka, are not computed because these parameters cease to be meaningful in the context of $\mathrm{H}_{2}$ flames that do not conform to the concept of stretched wrinkled thin flames. Other parameters listed in Table 1 pertain to the analysis of the flowfields in the next section.

\section{Flowfield Features}

Mean flowfield features from the measured 2D velocity vectors for case H-D $\left(U_{0}=15 \mathrm{~m} / \mathrm{s}\right)$ shown in Fig. 2 are similar to those reported for $\mathrm{CH}_{4}$ flames in similar conditions. A nearfield (i.e., near the LSB nozzle exit plane) uniform flow region is found at the center $(-10<r<10 \mathrm{~mm}$ ) flanked by the faster flow of the annular swirling jet (at $r= \pm 22$ $\mathrm{mm})$. Axial flow deceleration within the non-swirling center region is outlined by contours of the normalized axial velocity, $u_{z} / U_{0}$ at $z<15 \mathrm{~mm}$. The position of the local minima $(z \approx 15 \mathrm{~mm}$ and $r=0)$, marks the leading edge of the flame brush and is closer to the nozzle than it is for the corresponding $\mathrm{CH}_{4}$ flame (Case M-D), as reported in Day et al.[1]. As discussed in Cheng et al. [15], an upstream shift in the $\mathrm{H}_{2}$ flame positions relative to those of $\mathrm{CH}_{4}$ is a consequence of the higher $\mathrm{H}_{2}$ turbulent displacement flame speeds. Downstream of this minimum point, heat release reverses the deceleration trend and generates a slight mean acceleration. In the far field, $\mathrm{z}>100 \mathrm{~mm}$, the formation of a large but very weak central recirculation zone is outlined by the $u_{z}=0$ contour.

Fig. 3 shows the centerline profiles of the normalized mean axial velocity, $u_{z} / U_{0}$, and the normalized 2D turbulent intensity energy $q^{\prime} / U_{0}$. The $u_{z} / U_{0}$ preofiles deduced from the simulations are also shown in Fig. 3a. Differences between the simulated and experimental $u_{z} / U_{0}$ profiles are discussed later in this Section. The three experimental 
profiles of Fig. 3a illustrate that the decay of $u_{z} / U_{0}$ in the nearfield $(z<15 \mathrm{~mm})$ is linear and similar across the three flow rates. Slight differences between the profiles of $\mathrm{H}-\mathrm{C}$, and those at higher velocities suggest that the flowfield is still evolving with increasing $U_{0}$. Otherwise, the key features of the $u_{z} / U_{0}$ profiles and the nearfield self-similar behavior are typical of those measured in flames generated by the LSB. Listed in Table 1 are the two parameters that characterize the self-similar features of the nearfield central divergence zone: the normalized axial stretch rate, $a_{z}$ and the virtual origin $z_{o}$. These values, deduced according to the procedured described in [15], are essentially the same as those obtained for the $\mathrm{CH}_{4}$ flames. In Fig. 3b, the scaled turbulent intensities in the nearfield $(z<15 \mathrm{~mm})$ are the same level across the three flow rates as expected of turbulence produced by a perforated plate. The non-decaying nature of nearfield $q^{\prime} / U_{0}$ is also reported in previous papers $[1,12]$. As listed in Table 1 , the turbulent intensity produced by the perforate plate is anisotropic, with the longitudinal fluctuations $u_{z}$ ' about 50\% higher than the transverse fluctuations $u_{r}^{\prime}$. In the farfield, the $q^{\prime} / U_{0}$ profiles for the three flame are dissimilar due to the contributions from flame generated flow acceleration becoming proportionally smaller as $U_{0}$ is increased.

Following the procedure developed for the $\mathrm{CH}_{4}$ flames, mean flow velocity profiles were constructed from the 3D simulation data by transforming the velocity into polar coordinates and azimuthally averaging each snapshot of simulation data. The snapshot averages are then averaged over the simulated evolution time. In Figs. 4 and 5, the normalized 2D velocity vectors from PIV, and the azimuthally averaged results for the LMC simulations are compared for cases H-C and H-D. Contours of the normalized velocity vector magnitude, $|u| / U_{0}$, are shown in the background. The $T=350 \mathrm{~K}$ isotherms on the simulation results approximate the position of the leading edge of the flame brush in each case.

The PIV plots on the left sides of Figs. 4 and 5 show that the main difference between between H-C and H-D flames is an upstream shift in location and an enlargement of the recirculation zone in the farfield. Both are characterstics of an evolving flowfield. Otherwise, the overall flow pattern, the mean flame position and the flame shape are similar. The simulated results on the right sides of Figs. 4 and 5 verify that the LMC method captures the salient features of the flame and its flowfield. The simulated $\mathrm{H}_{2}$ flames are closer to the LSB nozzle than the simulated $\mathrm{CH}_{4}$ flames of Day et al. [1]. The upstream shift in LSB flame position when burning $\mathrm{H}_{2}$ is consistent with experimental observation [15]. The main difference between the the simulated and measured flames is seen in the farfield, where the size and shape of the recirculation zones of the simulated flames are broader and remain unchanged with increasing velocity. This indicates that the simulations capture the salient features of the fully developed flame flowfield. Because the central recirculation zones are very weak with large time and spatial scales, the length of the simulation may not be sufficiently long to capture the evolving features of these large, slowly-evolving flow structures.

In the nearfield, small discrepancies between the measurements and the simulations are shown by the overall flame shapes, flame lift-off height, and the magnitudes of the local velocities. The simulated flames positions are farther downstream than the experiments (also seen in Fig. 3a). In the swirling-flow regions, the magnitudes of the simulated peak velocities are slightly lower than the measurements. The values of the normalized stretch rate, $a_{z}$, and the axial location of the virtual origin, $z_{0}$, for the simulation are given in Table 1 along with the measured values. Near the LSB nozzle, the two simulated flows exhibit similar values for $a_{z}$, but both are slightly larger than those given by the experimental measurements. The virtual origin of both simulated flows are smaller than the experimental data. The differences are due to the radial flow divergence in the simulations occuring at positions farther downstream than the experiments as shown by the $u_{z} / U_{0}$ profiles of Fig. 3a . As discussed in Ref. [1], we attribute the observed discrepancies in mean flame statistics to a less-than-ideal characterization of the inlet velocity specification used for the simulations.

\section{Local flame analysis}

There are a variety of approaches to analyzing multidimensional transport and flow effects on the detailed structure of premixed flames. Clearly, any framework for this purpose must begin with the notion of a parcel of unburned fuel being converted to combustion products. What remains in such a picture is a control-volume analysis of the physical processes that take place during this conversion: advection, diffusion and chemical reactions. A popular approach is 
based on taking the boundaries of a control volume to be be defined by the integral curves of a scalar reaction progress measure. When such a measure is unique, the subsequent analysis is simplified because diffusive transport across the boundaries of the control volume vanishes. Moreover, if these structures persist over a finite time, the multidimensional problem reduces approximately to a steady 1D "flamelet" [35]. The flamelet concept has been the basic paradigm for experimental and theoretical premixed flame analysis. For example, it is common to base experimental data analysis on the notion that we only need to identify the flame location (using Mie scattering or PLIF data). The local flame structure is given then by the idealized 1D flames with possible corrections for steady stretch.

The conventional flamelet construction (see e.g., [3,4]) is made by starting with a tessellation of a suitably defined flame surface and then extending the bounds of a control volume along integral curves of the gradient of reaction progress, $c$, defined in terms of temperature (See Fig. 13 in Ref. [1]). The simulation or experimental data is collected over this path, and compared to profiles of representative 1D steady flames. Though providing useful information about the flame, this type of construction presents a flame-centric perspective that does not represent the fluid motion approaching the flame along the local flame coordinate. The time for a fluid parcel to move through the preheat zone and the reaction front is roughly comparable to the eddy turnover time of 3-4 ms in the LSB experiments. The conditions can therefore change significantly during the transit of a fluid element from the reactants to the products $[1]$.

Approaches based on scalar iso-surfaces encounter an additional set of issues in lean $\mathrm{H}_{2}$-air mixtures, where there is significant differential fuel transport. The issue is evident in the steady $1 \mathrm{D}$ flame profiles shown for an $\mathrm{H}_{2}$-air mixture at $\phi=0.37$ in Fig. 7a. Clearly, the temperature and $c$ (based here on concentration of $\mathrm{H}_{2}$ ) do not exhibit the linear dependence observed for lean $\mathrm{CH}_{4}$ cases. This suggests that there is no unique measure for instantaneous reaction progress in these flames, making identification of a flame contour problematic, even in 1D. The phenomenology of the 1D flame can be described as follows. As fluid parcels approach the flame, reactions deplete the fuel and oxidizer, driving a gradient in their respective concentrations that is co-aligned with the flow through the flame. Relative to the oxidizer, hydrogen has a much higher diffusivity, and therefore larger diffusion velocity in this zone. For a steady flame with constant atomic fluxes this leads to a local drop in the ratio of hydrogen to oxygen atoms. The atomic stoichiometry,

$$
\phi^{l}=\frac{\left(\mathcal{N}_{H} / \mathcal{N}_{O}\right)_{\text {local }}}{\left(\mathcal{N}_{H} / O\right)_{\text {stoich }}}
$$

expresses the local value of this ratio, normalized by its value in a stoichiometric mixture. Here, $\mathcal{N}_{n}$ is the atomic density of atom $n$, and the subscript stoich is the value of this ratio in a stoichiometric mixture. For the steady unstrained flame with inlet equivalence ratio, $\phi=0.37, \phi^{l}$ is shown in Fig. $7 \mathrm{a}$, and varies from 0.37 far from the flame to a minimum of 0.23 at $c=0.55$. In multiple dimensions, preferential diffusion distributes fuel normal to integral curves in the temperature, $T$. This leads to localized fuel-rich and fuel-depleted pockets, and significantly complicates the relationship between the various quantities typically used to measure reaction progress (see, for example, [3]). Even in this case, the flamelet construction may still be useful, but requires a more involved accounting of "transverse" transport (see, e.g., [36] and references cite therein for extensive developments in this direction). As a result, care must be exercised when interpreting instantaneous $\mathrm{H}_{2}$ and blended $\mathrm{H}_{2} / \mathrm{CH}_{4}$ flame simulations in terms of the steady flame idealization, particularly when attempting to infer cause-and-effect relationships.

An alternative simplified picture of the flame can be constructed by tracking a parcel of fluid in the Lagrangian frame as it passes through the reaction front. In this case, the advective transport through the control volume boundaries is identically zero, and what remains is an explicit accounting of the relative effects of diffusion and reaction chemistry. Importantly, this alternative picture does not rely on assumptions about the relative timescales of fluid motion and chemistry. Since it is clear that this diagnostic does not have an obvious experimental counterpart, it serves rather as an opportunity unique to detailed simulation that allows us to probe the cause and effect relationships of turbulence/chemistry interactions. The pathlines are generated by passively advecting particles with the fluid velocity during the simulations. In the Lagrangian frame, the mass fraction of fuel satisfies the following conservation equation:

$$
\frac{D Y_{H 2}}{D t}=\omega_{H 2} W_{H 2}-\frac{1}{\rho} \nabla \cdot \Gamma_{H 2}
$$

where $\mathrm{Y}_{\mathrm{H} 2}$ is the mass fraction of $\mathrm{H}_{2}, \rho$ is the fluid density, and $\omega_{H 2}$ and $\mathrm{W}_{H 2}$ are the molar chemical production rate and molecular weight of $\mathrm{H}_{2}$, respectively. Fig. 7b shows the varation of the chemical ("Reac") and diffusion ("dH $\mathrm{H}_{2}$ ") 
terms, the first and second terms on the right hand side of (1), respectively, as a function of progress, $c$, defined in terms of fuel mole fraction for the steady unstrained 1D flame. The dotted orange line, labeled "Adv", is the value of the Lagrangian derivative, $D Y_{H_{2}} / D t$. The diffusive flux divergence term, $\mathrm{dH}_{2}$, from the $1 \mathrm{D}$ idealization will be used in the following analysis as a basis to compare the behavior of the 3D turbulent flame system.

For the 3D time-dependent simulation data, initial seed points are chosen in the cold region slightly upstream of the flame, and as the Lagrangian paths are integrated in time, local properties of solution are collected. The main benefit of this approach is that the dynamic changes within the reacting fronts are incorporated explicitly, allowing us to explore the effects of the time-varying fields. Additionally, the pathline diagnostic can be applied without ambiguity to interrogate different regions of the flame including regions where reactions may not be complete, such as the local non-burning regions of the $\mathrm{H}_{2}$ flames and near the outer edges of the flame where significant air entrainment and dilution are expected. In the near unity Lewis number cases considered previously, we have shown that both the flamelet and pathline types of analysis yield similar results. Here, the pathline technique is applied to investigate the simulated results of $\mathrm{H}_{2}$ flames to explore the changes in local reactivity due to thermal/diffusive effects and to aid in the interpretation and analysis of the OH-PLIF measurements.

A set of 10,000 Lagrangian pathlines were extracted from the H-C and H-D flame solutions, that were seeded within the central region $(r<20 \mathrm{~mm})$ of the burner. Fig. 8 shows 300 randomly selected pathline trajectories in physical space for case H-D. They collectively outline the radially divergent nature of the flow. A typical length of these pathlines is on the order of $10 \mathrm{~mm}$, which, as in pathlines from $\mathrm{CH}_{4}$ flame solutions, represents the convective distance the particles move as they react from cold fuel to hot products. "Hot products" is defined here where $c$, based on $\mathrm{H}_{2}$ concentration, assumes values larger than 0.99 . The main difference between Fig. 8 for a $\mathrm{H}_{2}$ flame and Fig. 14 of Day, et al. for a $\mathrm{CH}_{4}$ flame is that the temperatures at the end points of the $\mathrm{H}_{2}$ pathlines are non-uniform. This issue will be elaborated further in the next section. Properties extracted on the pathlines include temperature, velocity components, species concentrations, and chemical production rates. In addition, we compute local mean curvature, $M=\kappa_{1}+\kappa_{2}$ (where $\kappa_{i}$ are the principle curvatures of the local temperature isosurface), Gaussian curvature, $G=\kappa_{1} \cdot \kappa_{2}$, and convective and diffusive flux divergences, and the cumulative $\mathrm{H}_{2}$ consumption, $\mathrm{H}_{2}^{\text {con }}$,

$$
\mathrm{H}_{2}^{\mathrm{con}}(s)=\frac{\int_{s(c=0)}^{s} \omega_{H_{2}} d s}{\int_{s(c=0)}^{s(c=1)} \omega_{H_{2}} d s}
$$

where $\omega_{H_{2}}$ is the local chemical consumption rate of $\mathrm{H}_{2}$ in moles / (volume time), and $s$ is the arclength along the Lagrangian pathline. We also compute $\omega^{\max }$, the normalized fuel consumption peak, $\omega^{\max }=\omega_{\mathrm{H}_{2}}^{\max } / \omega_{\mathrm{H}_{2}-1 D}^{\max }$, where $\omega_{H_{2}}^{\max }$ is the maximum instantaneous $\mathrm{H}_{2}$ consumption rate along each pathline, and the normalization $\omega_{H_{2}-1 D}^{\max }$ is the corresponding value of $\omega^{\max }$ from the 1D flat flame solution. Due to the decoupling of temperature and reaction progress, the local tangential strain rate (or, divergence of the flow in the $c$ contours) is not particularly useful and so is not presented.

As an aside, we comment here on our choices of progress measures that are implicit in the above definitions of $c$ and local curvatures, $\kappa_{i}$. As previously discussed, multidimensional lean hydrogen flames do not admit a unique parameterization of flame progress, yet for the present discussion we will make use of such a notion in order to normalize the relative Lagrangian positions of the fluid parcels. Two obvious candidates are the temperature and fuel concentration fields, but each has a fundamental limitation. The temperature field is problematic as a measure of reaction progress; due to local hot spots, the temperature of a parcel can be nonmonotonic. On the other hand, we wish to monitor the local curvature of the reaction zone near the flame surface, but this is precisely where the fuel concentration is vanishing; the curvatures of the fuel isodensity surfaces near vanishing concentrations are poorly behaved. As a result, while the concentration of fuel serves as a natural measure of reaction progress, the isotherms are used here to quantify the local flame surface curvature. Note that without these assumptions, it is impossible to relate the Lagrangian statistics to the traditional notion of a flame.

Fig. 9 shows the joint probability density functions (JPDF) of $\mathrm{H}_{2}^{\text {con }}$ with mean curvature ( top row) and with $\omega^{\max }$ ( bottom row). Consistent with the analysis of $\mathrm{CH}_{4}$ flames, the mean curvature computed at $c=0.8$ is used as the basis of comparison. But as seen in Fig. 7, this $c$ value does not correspond to the maximum consumption rate in the solution of a $1 \mathrm{D}$ unstretched laminar $\mathrm{H}_{2}$-air flame at $\phi=0.37$, as it did for the referenced $\mathrm{CH}_{4}$ cases. 
Starting with Figs. 9a and 9b, a strong correlation between $\mathrm{H}_{2}^{\text {con }}$ and curvature is clearly shown for both flames. The high $\mathrm{H}_{2}^{\text {con }}$ pathlines experience positive curvature (convex to the reactants) and the low $\mathrm{H}_{2}^{\text {con }}$ pathlines experience negative curvature (concave to the reactants). The effect of increased turbulence intensity, however, is not obvious. It is manifested by slight broadening of the JPDF contours of Case H-D (Fig. 9b). The most probable values of $\mathrm{H}_{2}^{\text {con }}$ for both flames are the same with $\mathrm{H}_{2}^{\text {con }}=1.6$ at mean curvature of $2501 / \mathrm{m}$. The non-burning pathlines for both cases where $\mathrm{H}_{2}^{\text {con }} \approx 0$ spread over a range of negative curvatures from 0 to over $-40001 / \mathrm{m}$. The overall shape of the JPDF of $\mathrm{H}_{2}^{c o n}$ with mean curvature is consistent with the JPDF of the normalized local consumption flame speed, $S_{c} / S_{L}$ with curvature, observed for lean hydrogen flames in Fig. 10 (a) of Ref. [4]. The largest $\mathrm{H}_{2}^{\text {con }}$ values of 5, attained respectively for both Cases H-C and H-D, are also consistent with the largest values of $S_{c} / S_{L}$ from the iso-surface analysis. Therefore, the $\mathrm{H}_{2}^{\text {con }}$ computed on the pathlines is considered an analog of $S_{c} / S_{L}$ quantity computed for a unity Lewis number fuel.

Both JPDFs of $\mathrm{H}_{2}^{\text {con }}$ with $\omega^{\max }$ on Fig. $9 \mathrm{c}$ and $9 \mathrm{~d}$ have their maxima at the origin meaning that pathlines with no $\mathrm{H}_{2}$ consumption and zero $\mathrm{H}_{2}$ reaction rates are highly probable. These non-burning or very weak burning regions were excluded from the iso-surface analysis of $S_{c} / S_{L}$ in Ref. [4] because there was no unique definition of a flame front. However, these regions can be examined via the pathline diagnostics without ambiguity. To gain insights into the local flame structures and scalar properties at different burning regions of the $\mathrm{H}_{2}$ flames, the JPDF of $\mathrm{H}_{2}^{\text {con }}$ with $\omega^{\max }$ is used to identify five groups of pathlines, A-E, of similar properties. Division into pathline groups follows the approach of Bell, et al. [37]. In addition to the strong, intermediate and weak burning regions used in the reference, we add two others to account for the higher-turbulence conditions here: very-strong and very-weak burning. Table 2 lists the $\mathrm{H}_{2}^{\text {con }}$ and $\omega^{\max }$ values that define the groups. Note that these bounds are arbitrary and do not provide complete coverage of the conditions. Approximately 300 to 400 pathlines are extracted at random from each of the five groups for analysis. The intermediate burning group includes the local peak of the JPDFs for $\mathrm{H}_{2}^{c o n}$ with $\omega^{\max }$, and corresponds also to the most probable $\mathrm{H}_{2}^{\text {con }}$ consumption value on the JPDFs of $\mathrm{H}_{2}^{\text {con }}$ with mean curvature.

Table 2: Criteria to define pathline groups for the simulated $\mathrm{H}_{2}$-air flames

\begin{tabular}{|c|c|c|c|}
\hline Group & $\omega^{\max }$ & AND & $\mathrm{H}_{2}^{\text {con }}$ \\
\hline A: Very strong burning & $8.2-8.6$ & & $2.0-2.4$ \\
\hline B: Strong burning & $7.4-7.8$ & & $1.8-2.2$ \\
\hline $\mathrm{C}$ : Intermediate burning & $5.4-5.8$ & & $1.4-1.8$ \\
\hline D: Weak burning & $2.5-2.9$ & & $0.8-1.2$ \\
\hline E: Very weak burning & $0.2-0.3$ & & $0.1-0.3$ \\
\hline
\end{tabular}

\section{Flame Front Structures}

Fig. 10 compares the mean local atomic stoichiometry, $\phi^{l}$, computed for the five groups in terms of reaction progress, $c$. The error bars are the corresponding RMS values in each group. At $c<0.2$ the values of $\phi^{l}$ for all are close to the value from the 1D laminar flame calculation. As the reactions proceed, $\phi^{l}$ in these groups increases above the 1D laminar flame levels to indicate local fuel enrichment. The higher turbulence in case H-D does not correspond to an observable increase in $\phi^{l}$ compared to case $\mathrm{H}-\mathrm{C}$.

Fig. 11 shows the temperature profiles along pathlines for the very-strong, intermediate and very-weak groups. The profiles are plotted in terms of $s$, the distance on the pathline with the origin shifted to the $c=0.8$ location. For reference, the figure includes the temperature profiles from the steady unstretched laminar 1D flame solution at $\phi=0.37$. For $\mathrm{H}-\mathrm{C}$ at $U_{0}=10 \mathrm{~m} / \mathrm{s}$, the profiles from the very-strong (Fig. 11a) and intermediate (Fig. 11b) burning pathlines show superheating (above the peak temperature of the steady flame) due to $\mathrm{H}_{2}$ enrichment; the temperature attained at $s=0$ (i.e. $c=0.8$ ) can be $100 \mathrm{~K}$ to $300 \mathrm{~K}$ higher than the adiabatic flame temperature of $1360 \mathrm{~K}$. Otherwise, these profiles generally follow the unstrained 1D laminar flame profile with some broadening of the preheat zone. The 
temperature profiles in the very-weak burning group of case H-C (Fig. 11c) are quite different due to preheat zone broadening and underheating at $s>0$. The highest temperatures attained on some of the very weak burning pathlines can be $300 \mathrm{~K}$ lower than the adiabatic flame temperature. Fuel depletion along theses pathlines can be seen by the low values of $\phi^{l}$. Whether or not burning occurs will be examined later in this section. For case H-D (Figs. 11d-f), similar trends of super- and under-heating in the three groups are also shown. The overall effect of higher turbulence is a slight broadening of the reaction fronts of the very weak burning group. These temperature profiles show that the local peak temperatures in different pathline groups can vary by up to $600 \mathrm{~K}$.

The local diffusive flux divergence of $\mathrm{H}_{2}, \mathrm{dH}_{2}$ on the Lagrangian paths is shown in Fig. 12. The plots are colored by contours of the local mean curvatures. As expected, the very-strongly burning pathlines of both flames (Fig. 12 a $\&$ d) have positive curvatures; the reaction fronts are convex to the reactants. Relative to the diffusive flow of fuel in the 1D flame, there is a significantly larger source of $\mathrm{H}_{2}$ into the pathlines from diffusion that occurs at $c>0.6$. Note that $\mathrm{dH}_{2}$ for $c<0.6$ is slightly more negative in both flames than in the 1D flame profile, indicating weak transport of $\mathrm{H}_{2}$ out of the pathlines and resulting in a corresponding decrease in $\phi^{l}$ between $0<c<0.6$ on Fig. 10. For the intermediate burning group (Fig. 12 b \& e), mean curvatures are lower than those of the very-strongly burning paths. Most pathlines have positive mean curvature but many are slightly negative. The rates of $\mathrm{H}_{2}$ diffusing into the intermediate burning pathlines at $c>0.6$ are smaller than those in the very-strong burning group. The trend is consistent with a general correlation of $\omega^{\max }$ vs. mean curvature as shown by the JPDFs of Fig. $9 \mathrm{c} \& \mathrm{~d}$. The mean curvatures of the very-weak burning pathlines (Fig. $12 \mathrm{c} \&$ f) are predominantly negative, showing that the isotherm used as a surrogate for the reaction front is convex to the reactants in these regions. The value of $\mathrm{dH}_{2}$ throughout these pathlines is less than that of the 1D flame for any value of $c$. Integrated across the entire flame, this indicates that the lower temperature observed in the cusps of $\mathrm{H}_{2}$ flame is due to depletion of fuel by differential diffusion to neighboring pathlines. There are, however, some overlaps in the range of mean curvatures of the intermediate and very-weak burning groups. The implication is that the reaction front topology captured by 2D laser diagnostics (i.e. the sign of the curvatures of the captured wrinkled geometry) does not provide adequate information to infer the local burning structure and state.

Temperature on the pathlines is plotted against $c$ in Fig. 13, and shows significant deviations from the 1D laminar flame profile. In the very-strong and intermediate burning groups of cases H-C and H-D (Fig. 13 a,b,d \& e), the profiles are almost linear between $0.2<c<0.9$ reaching the level of the adiabatic flame temperature at $c=0.9$. Super-heating occurs at $c>0.9$. In the very-weak burning group (Figs. $13 \mathrm{c} \& \mathrm{f}$ ), the temperatures are significantly lower than in the other groups, and are closer to the 1D flame calculation between $0.2<c<0.8$. That the temperatures at lower $c$ in the very-weak burning group are higher than the $1 \mathrm{D}$ flame calculation seems to imply diffusive heat transport. Negative values of $\mathrm{dH}_{2}$ here again indicates that fuel depletion is associated with a temperature profile that drops below the laminar flame curve at $c>0.8$.

Fig. 14 shows the pathline profiles of normalized $\mathrm{H}_{2}$ consumption rate, $\omega=\omega_{H_{2}} / \omega_{H_{2-1 D}}^{\max }$, compared with the 1D laminar flame solution. Recalling that $\omega^{\max }$ is a parameter that defines the various pathline groups, the peaks of these profiles correspond to the range of values listed in Table 2. It is apparent that the $\omega$ profiles are not consistent with the 1D flame. Whereas $\mathrm{H}_{2}$ consumption occurs at $c>0.6$ on the 1D laminar flame profile and peaks at $c=0.9, \mathrm{H}_{2}$ consumption in the very-strong and intermediate burning paths (Figs. $14 \mathrm{a}, \mathrm{b}, \mathrm{d} \& \mathrm{e}$ ) starts at $c=0.2$ and peaks near $c=0.8$. Peak $\mathrm{H}_{2}$ consumption rates of all but the very-weak pathlines are near $c=0.8$ where fuel enrichment, shown by the color contours of $\mathrm{H}_{2}$ diffusion parameter, $\mathrm{dH}_{2}$, is also at its highest level. In the very-weak burning group (Figs. $14 \mathrm{c} \& \mathrm{f}$ ), significant fuel depletion results in low $\mathrm{H}_{2}$ consumption. These pathlines consume 10 times less fuel compared to those in the very-strong burning group.

\section{Simulated and experimental flame curvatures}

For near-unity Lewis number flame such as $\mathrm{CH}_{4}$, the morphology of the experimental flames was inferred from extracting flame edges from OH-PLIF images in terms of a mean progress variable, $\bar{c}$, and the 2D local flame curvature $\kappa$. To derive these properties for $\mathrm{H}_{2}$ flames in the presence of gaps in the OH-PLIF images, we utilize the simulation 
results to assist in the development of a new analysis method that will produce physically meaningful results. Fig. 15 shows $\mathrm{OH}$ mole fraction, $\mathrm{X}_{\mathrm{OH}}$, in terms of the distance on the pathline, $s$. In the very-strong and intermediate groups (Figs. 15 a,b,d \& e), $\mathrm{X}_{\mathrm{OH}}$ can be more than six times higher than $\mathrm{X}_{\mathrm{OH}}$ in the $1 \mathrm{D}$ laminar flame. Conversely, $\mathrm{X}_{\mathrm{OH}}$ in the very-weak group (Figs. $15 \mathrm{c} \& \mathrm{f}$ ) is lower than the 1D flame. The increase in peak $\mathrm{X}_{\mathrm{OH}}$ from the very weak burning group to the very strong burning groups is consistent with the strong correlation between $S_{c} / S_{L}$ and peak $\mathrm{X}_{\mathrm{OH}}$ reported in Day et al. [4]. The gradient of the $\mathrm{X}_{\mathrm{OH}}$ profiles along the pathlines, $\partial X_{O H} / \partial s$, is also steeper for the very-strong and intermediate burning groups than for the very-weak burning groups. This means that the gradient of the intensity signal $\mathrm{I}_{\mathrm{OH}}$ captured by OH-PLIF can likely also be used as a criterion to distinguish between the non-burning and burning regions in experiments. Based on this observation a method has been developed to filter the edges obtained by applying a simple threshold criterion on OH-PLIF images. As discussed later in this section, this method can distinguish leading and and trailing edges to allow the analysis of $\mathrm{H}_{2}$ flame curvature for cases $\mathrm{H}-\mathrm{C}$ and H-D .

The computation of a mean progress variable, $\bar{c}$, for the turbulent flame brush, which is is trivial for $\mathrm{CH}_{4}$ flames via a simple binarized procedure (see Fig. 6 middle image where black and white correspond respectively to $c=0$ and 1), is also problematic due to the gaps on the OH-PLIF images of $\mathrm{H}_{2}$ flames. Here we develop an alternative approach that examines the correlation of $\mathrm{OH}$ concentration with parameters that can be used to define a more relevant measure of mean reaction progress, $\bar{c}$. The $\mathrm{X}_{\mathrm{OH}}$ profiles in Fig. 15 are colored by the percentage of $\mathrm{H}_{2}$ remaining on the pathlines. For all regions, the color indicates that over $95 \%$ of $\mathrm{H}_{2}$ is gone when $\mathrm{X}_{\mathrm{OH}}$ reaches its local peak. The significant implication is that the $\mathrm{OH}$ signals detected by OH-PLIF, regardless of their intensities (particularly in the cusps region with very low levels of $\mathrm{OH}$ ), corresponds to locations where the fuel concentration is near zero. Note that one interpretation of $\bar{c}$ is the statistical probability of finding combustion products at a given location within the flowfield (or, 1 minus the statistical probability of finding the fuel). It follows that all locations on the OH-PLIF images with nontrivial $\mathrm{OH}$ signal effectively correspond to regions with no remaining fuel (i.e. $c=1$ ). However, due to the significant dropout and unevenness of the $\mathrm{OH}$ signal intensity on the products side of the flame, applying this methodology in a consistent way with acceptable uncertainties requires the development of new image processing algorithms, and will be pursued in future work.

Fig. 16 shows the steps in the analaysis of an OH-PLIF image to extract the reaction fronts for curvature calculation. Details of the analysis and the uncertainties in the results can be found in Appendix A. The analysis begins by normalizing the individual raw OH-PLIF image by a maximum intensity and applying an anisotropic diffusion process describe by Perona and Malik [38] to remove pixel noise without smearing the gradients (Fig. 16a). An edge-finding algorithm is then used to locate the contours of the $\mathrm{OH}$ signal at a threshold value of $30 \%$ of the peak $\mathrm{I}_{\mathrm{OH}}$. This generates the contour islands as seen at the lower right frame of Fig. 6. These islands outline the regions of high $\mathrm{OH}$ concentration, as well as their 'back side' where the $\mathrm{OH}$ concentration falls off. In a separate process, the laser-beam corrected images undergo a $5 \times 5$ pixel Gaussian smoothing prior to the calculation of local intensity gradient, i.e. $\left|\nabla \mathrm{I}_{\mathrm{OH}}\right|$, where $\mathrm{I}_{\mathrm{OH}}$ is the pixel intensity. The result is shown in grayscale on Fig. 16b. We assume that the regions of high $\left|\nabla \mathrm{I}_{\mathrm{OH}}\right|$ are associated with regions of high reactivity, and filter the grayscale $\left|\nabla \mathrm{I}_{\mathrm{OH}}\right|$ image using a threshold corresponding to $25 \%$ of the peak $\left|\nabla \mathrm{I}_{\mathrm{OH}}\right|$ value from the experiments. This generates a binary image marking the locations where burning takes place. The $\left|\nabla \mathrm{I}_{\mathrm{OH}}\right|$ binary image is shown in green on Fig. 16c together with the $\mathrm{OH}$ contours shown in red. It can be seen that the backside of the $\mathrm{OH}$ edges are excluded from the $\left|\nabla \mathrm{I}_{\mathrm{OH}}\right|$ regions. The product of the binary edge and the binary $\left|\nabla \mathrm{I}_{\mathrm{OH}}\right|$ images then defines the conditioned broken flame edges shown in Fig. $16 \mathrm{~d}$. The $2 \mathrm{D}$ curvature, $\kappa_{2 D}$, of these broken edges is computed in the usual way by

$$
\kappa_{2 D}=\frac{x^{\prime} y^{\prime \prime}-y^{\prime} x^{\prime \prime}}{\left(x^{2}+y^{2}\right)^{3 / 2}}
$$

The sign convention for $\kappa_{2 D}$ is that is positive when the center of curvature lies on the products side of the flame.

To be consistent with the analysis of the OH-PLIF results, the mean curvatures from the simulations are also filtered using a threshold criterion based on $\left|\nabla \mathrm{X}_{\mathrm{OH}}\right|$. Examination of the scatter plots for mean curvature, $\mathrm{H}_{2}^{\text {con }}$, and $T_{c=0.99}$, (i.e. temperature on pathlines at $c=0.99$ ) led to the choice of $\left|\nabla \mathrm{X}_{\mathrm{OH}}\right|_{\max }>0.3 \times\left|\nabla \mathrm{X}_{\mathrm{OH}}\right|_{\max -1 \mathrm{D}}$ as a filter threshold. This filters out all pathlines with zero fuel consumption, and most but not all pathlines in the very-weak 
burning regions. The justification for this filtering process and the sensitivity of the results to the threshold value are discussed in Appendix B. Fig. 17 shows the JPDF of $\omega_{\max }$ with $T_{c=0.99}$ before and after filtering. The main effect of the filter is to eliminate (nonburning) pathlines from the analysis that do not attain temperatures above $1200 \mathrm{~K}$ until well after the fuel is fully depleted. This criterion is less rigid than the $S_{c} / S_{L}>0.35$ cutoff used in Day et al. [4], and is consistent with the physics of the burning regions shown on the pathline profiles.

The PDFs of the filtered and unfiltered $\kappa_{2 D}$ for the two simulated flames are compared in Fig. 18 with those obtained from the OH-PLIF measurements. All PDFs peak at $\kappa_{2 D}>0$, which indicates that burning takes place mostly in convex regions. Filtering the simulated results lowers the probability of flame fronts with large negative curvatures, but the process has no effect on the location of the peak. Consistent with the generation of finer scale flame wrinkles at higher turbulence, broadening of the PDFs with increasing $U_{0}$ is observed for both simulations and measurements. Though the curvature pdfs from the simulations are consistent with the corresponding pdfs from measurements, further interpretation and analysis of the results should consider the differences between the natures of 2D (measurements) and 3D (simulation) curvatures.

\section{Conclusion}

We have presented simulations of laboratory-scale LSB flames in order to develop a characterization of the interaction of thermal/diffusive unstable flames with turbulence at the correct scales of laboratory experiments. The simulations were conducted using a block-structured adaptive low Mach reacting flow solver that has a unique capability to incorporate a large simulation domain while simultaneously including a detailed model of hydrogen combustion that includes differential species transport and kinetic reactions. We demonstrated that the global features of the simulation flames, in terms of mean shape and position above the burner, were consistent with corresponding experimental measurements.

The detailed simulation results were probed using a number of diagnostic techniques based on Eulerian and Lagrangian diagnostic approaches. We discussed the pitfalls of traditional Eulerian analysis techniques when applied to cellular flame systems such as the difficulty of defining an appropriate measure of "flame progress". The issues related to defining a flame surface that forms the basis for an Eulerian analysis become particularly difficult in regions where there is little or no burning. In this case, different potential choices for a flame surface become highly decorrelated (see Fig. 3 in Ref. [3]) and are unrelated to any significant reaction processes. In addition, traditional Eulerian analysis techniques are based on a static picture of the flow field that implicitly assumes flow time scales are slow relative to flame time scales.

In view of these issues, the Lagrangian pathline approach is particularly appealing since there is no need to identify a flame surface and no inherent assumptions about these time scales. Changes in composition in a fluid parcel are decomposed into diffusive transport with neighboring fluid elements and reaction processes within the parcel without introducing any additional approximation. We note that the Lagrangian diagnostic does not have an experimental counterpart, and thus serves as an opportunity unique to detailed simulation that allows us to probe the cause and effect relationships of the turbulence/chemistry interactions with a perspective that is complementary to more traditional Eulerian analysis.

Statistics were collected in the Lagrangian frame for pathlines that moved through the entire range of burning zones in the cellular flames, allowing us to quantify the effects of differential diffusion in terms of local depletion and enhancement of fuel, and resulting increases in flame stoichiometries and post-flame exhaust temperatures. Along the pathlines, we recorded the chemical state and properties of the local flame surface (e.g. isotherm). For each flame, we divided the pathlines into 5 groups, based on the rate of fuel consumption experienced by each as they passed through the flame zone. We found that an integrated measure of consumption along the pathline can serve as the generalized analog of the Eulerian-computed consumption-based burning speed. We also computed the instantaneous diffusive fuel flux divergence along the pathlines, and showed a direct correlation between this and the integrated consumption rate. In order words, the diagnostic is able to quantify the direct effects of differential fuel diffusion, and explain 
the resulting increases in local fuel stoichiometry that lead to large variabilities observed in these flames along the reaction front.

Insights gained through the Lagrangian diagnostic analysis served as the underpinning of a new procedure to interpret OH-PLIF images from the LSB experiment. An edge-finding algorithm was described that was able to distinguish the leading and trailing edges of the flame zone, and to exclude non-burning portions of the domain based on a more complete understanding of the cellular burning structures. As a result, this new diagnostic is able to provide a more physically meaningful approximation to the "flame surface area" than traditional approaches based on PIV processing.

\section{Acknowledgement}

This material is based upon work supported by the U.S. Department of Energy, Office of Science, Office of Advanced Scientific Computing Research, Applied Mathematics program under contract number DE-AC02005CH11231. The simulations and analysis were performed on the Franklin and Hopper machines at NERSC under an INCITE award. The authors gratefully acknowledge Gary Hubbard in the LBNL Combustion Laboratory for assistance in data analysis.

\section{References}

\section{References}

[1] Day M, Tachibana S, Bell J, Lijewski M, Beckner V and Cheng R K 2012 Combust. Flame 159 275-290

[2] Bell J B, Cheng R K, Day M S and Shepherd I G 2007 Proc. Combust. Inst. 31 1309-1317

[3] Day M, Bell J, Bremer P T, Pascucci V, Beckner V and Lijewski M 2009 Combustion and Flame 156 1035-1045

[4] Day M S, Bell J B, Cheng R K, Tachibana S, Beckner V E and Lijewski M J 2009 J. Phys: Conference Series 180012031

[5] Day M S, Gao X and Bell J B 2011 Proc. Combust. Inst. 33 1601-1608

[6] Bell J B, Cheng R K, Day M S and Shepherd I G 2007 Proc. Combust. Inst. 31 1309-1317

[7] Zeldovich Y B 1944 Theory of Combustion and Detonation in Gases (in Russian) (Acad. Sci. USSR)

[8] Markstein G H 1949 The Journal of Chemical Physics 17 428-429

[9] Bregeon B, Gordon A S and Williams F A 1978 Combustion and Flame 33 33-45

[10] Mitani T and Williams F A 1980 Combust. Flame 39 169-190

[11] Baum M, Poinsot T J, Haworth D C and Darabiha N 1994 J. Fluid Mech. 281 1-32

[12] Bedat B and Cheng R K 1995 Combustion and Flame 100 485-494

[13] Cheng R K, Yegian D T, Miyasato M M, Samuelsen G S, Pellizzari R, Loftus P and Benson C 2000 Proc. Comb. Inst. 28 1305-1313

[14] Petersson P, Olofsson J, Brackman C, Seyfried H, Zetterberg J, Richter M, Aldén M, Linne M A, Cheng R K, Nauert A, Geyer D and Dreizler A 2007 Appl. Optics 46 3928-3936 
[15] Cheng R, Littlejohn D, Strakey P and Sidwell T 2009 Proc. Combust. Inst. 32 3001-3009 lBNL

[16] Bell J B, Day M S, Grcar J F and Lijewski M J 2006 A computational study of equivalence ratio effects in turbulent, premixed methane-air flames Proceedings European Conference on Computational Fluid Dynamics ed Wesseling P, Onate E and Periaux J European Community on Computational Methods in Applied Sciences (ECCOMAS) (TU Delft) cD-ROM ISBN 90-9020970-0

[17] Day M S and Bell J B 2000 Combustion Theory and Modelling 4 535-556

[18] Li J, Zhao Z, Kazakov A and Dryer F L 2004 International Journal of Chemical Kinetics 36 566-575 ISSN $1097-4601$

[19] Rehm R G and Baum H R 1978 J. of Research of the National Bureay of Standards 83 397-308

[20] Majda A and Sethian J 1985 Combustion Science and Technology 42 185-205

[21] Almgren A S, Bell J B and Szymczak W G 1996 SIAM Journal on Scientific Computing 17 358-369

[22] Nonaka A, Bell J B, Day M S, Gilet C, Almgren A S and Minion M L 2012 Combustion Theory and Modelling $161053-1088$

[23] Ern A and Giovangigli V 1998 Combust. Theory Modelling 2 349-372

[24] Grcar J F, Bell J B and Day M S 2009 Proceedings of the Combustion Institute 32 1173-1180

[25] de Charentenay J and Ern A 2002 Combust. Theory Modelling 6 439-462

[26] Ern A and Giovangigli V 1994 Multicomponent Transport Algorithms (Lecture Notes in Physics vol m24) (Berlin: Springer-Verlag)

[27] Nogenmyr K J, Petersson P, Bai X, Nauert A, Olofsson J, Brackman C, Seyfried H, Zetterberg J, Li Z, Richter M, Dreizler A, Linne M and Aldén M 2007 Proc. Combust. Inst. 31 1467-1475

[28] Nogenmyr K J, Bai X, Fureby C, Petersson P, Collin R, Linne M and Aldén M 2008 A comparative study of les turbulent combustion models applied to a low swirl lean premixed burner AIAA Aerospace Sciences Meeting vol Paper 2008-0513 (Reno, Nevada: AIAA)

[29] Nogenmyr K J, Fureby C, Bai X, Petersson P, Collin R and Linne M 2009 Combustion and Flame 156 25-36

[30] Taylor G I 1938 Proc. R. Soc. London, Ser. A 164 476-490

[31] Aspden A, Day M and Bell J 2011 Proc. Combust. Inst. 33 1463-1471

[32] Bell J, Cheng R, Day M, Beckner V and Lijewski M 2008 J. Phys: Conf. Ser. 125012027

[33] Cheng R K, Shepherd I G, Bedat B and Talbot L 2002 Combust. Sci. Technol. 174 29-59 Engineering, Computing \& Technology (ENGI)JAN

[34] Johnson M R and Cheng R K 2003 Dynamics of the flame flowfields in a low-swirl burner 19th International Colloquium on the Dynamics of Explosion and Reactive Systems (Hakone, Japan) LBNL 53405

[35] Peters N 1983 Combust. Sci. Technol. 30 1-17

[36] Goey L P H d and ten Thije Boonkkamp J H M 1999 Combust. Flame 119 253-271

[37] Bell J, Day M, Gao X and Lijewski M 2010 SciDAC 2010 Conference Proceedings 149-153 chatanooga, TN

[38] Perona and J J M 1990 IEEE Trans. Pattern Anal. Mach. Intell. 12 629-639 


\section{Appendix A Reacting front detection from OH-PLIF images}

The computation of the reacting front curvature PDFs from OH-PLIF images of $\mathrm{H}_{2}$ flames involves a three step process. The first step is to locate the high-intensity $\mathrm{OH}$ contours using an edge finding algorithm. The second step is to mark the regions with large intensity gradients for use as a conditioning filter. The third step is a cross-product of the results from the first two steps to retain the burning edges and trim the segments that are not associated with the burning regions (i.e. the non-burning regions and the spurious edges in the products regions). To outline the $\mathrm{I}_{\mathrm{OH}}$ contours, the images are smoothed by an anisotropic diffusion algorithm, which is a locally adaptive nonlinear process that eliminates the noise on digital images without blurring the edges [38]. The filter is performed with the parameters $N_{\text {iter }}=10, \Delta t=1 / 7, \kappa=100$ and $C_{\text {cond }}=1 /\left(1+(\nabla I / \kappa)^{2}\right)$. After smoothing, the $\mathrm{I}_{\mathrm{OH}}$ contours are extracted using an algorithm identical to the one for $\mathrm{CH}_{4}$ flames [1]. The contours are defined by a threshold at $30 \%$ of the maximum intensity in each image. The threshold value was chosen so that it can be applied uniformly to all cases in our experimental data sets consisting of sixteen $\mathrm{CH}_{4}, \mathrm{H}_{2}$ and blended $\mathrm{CH}_{4} / \mathrm{H}_{2}$ flames. When applied to $\mathrm{CH}_{4}$ flames, the $30 \%$ threshold outlines contours that are within the preheat zones of the reacting fronts. For $\mathrm{CH}_{4}$ flames, the local $\mathrm{I}_{\mathrm{OH}}$ peak intensities and its gradients, $\left|\nabla \mathrm{I}_{\mathrm{OH}}\right|$, on the reacting fronts are uniformly high and the sensitivity of the $\mathrm{CH}_{4}$ curvature PDF's to the choice of the $\mathrm{I}_{\mathrm{OH}}$ threshold value defining the contours is small. For $\mathrm{H}_{2}$ flames, the local peak intensities and gradients vary but those associated with the burning regions remain high. Therefore, the uncertainties in the curvatures of the $\mathrm{H}_{2}$ reacting fronts, once extracted, are comparable to those from the $\mathrm{CH}_{4}$ flames.

The uncertainties in the curvature PDF are associated with the threshold value chosen to mark the regions of high $\left|\nabla \mathrm{I}_{\mathrm{OH}}\right|$ for the purpose of filtering and trimming the contours. Since OH-PLIF is not a quantitative diagnostics, guidance on selecting a meaningful cutoff $\left|\nabla \mathrm{I}_{\mathrm{OH}}\right|$ value is lacking. Therefore, we investigated how variation in the $\left|\nabla \mathrm{I}_{\mathrm{OH}}\right|$ threshold values may effect the filtering process. To reduce the noise from the $\left|\nabla \mathrm{I}_{\mathrm{OH}}\right|$ results, the OH-PLIF images were smoothed using a conventional 5 by 5 Gaussian filter prior to the computation of $\left|\nabla \mathrm{I}_{\mathrm{OH}}\right|$. Next the sets of grayscale $\left|\nabla \mathrm{I}_{\mathrm{OH}}\right|$ images for the three experimental flames were binarized by using intensity $\left|\nabla \mathrm{I}_{\mathrm{OH}}\right|$ thresholds with values ranging from $21 \%$ to $29 \%$ (at $1 \%$ intervals) of the maximum $\left|\nabla \mathrm{I}_{\mathrm{OH}}\right|$ values determined from the image sets. The $\mathrm{I}_{\mathrm{OH}}$ contours were then filtered by the sets of binarized $\left|\nabla \mathrm{I}_{\mathrm{OH}}\right|$ images. Because visual inspection of large sets of the filered $\mathrm{I}_{\mathrm{OH}}$ edges is impractical, we first examined the changes in the number of edge segments per image with the $\left|\nabla \mathrm{I}_{\mathrm{OH}}\right|$ threshold values. The results for cases H-C, H-D and H-E are shown in Fig. A-1. Due to the effect of turbulence, the number of segments increases with increasing velocity, meanwhile, the number of segments for different threshold values in the velocity condition is insensitive to the threshold value. Next we performed closer inspections on the filtered edge results obtained within the range where the number of segments are less sensitive to the $\left|\nabla \mathrm{I}_{\mathrm{OH}}\right|$ threshold values. An example of the composite image for case $\mathrm{H}-\mathrm{D}$ at a threshold of $25 \%$ is shown in Fig. 16 (c) where the edges (red) and gradient (green) are overlaid with the OH-PLIF image. It is evident that a 25\% threshold cutoff is effective in trimming spurious edges and gives satisfactory cutoffs in the transition regions where $\mathrm{OH}$ intensities start to decrease and cause the contours of loop to the backside to form islands. We also noticed that the truncation of the relative flat contours in the "fjord-like" deep cusps are most sensitive to the gradient threshold value. However, their contributions seem to be small. As shown in Fig. A-2, changing the threshold from 21\% to 29\% does not have a significant effects on the curvature PDF for case H-D. Therefore, the curvature PDFs in Fig. 18 were obtained by using a $25 \%$ gradient threshold.

\section{Appendix B Appendix B - Filtering of pathlines curvatures}

The scatter plots of mean curvature, $\mathrm{H}_{2}^{c o n}$, and $T_{c=0.99}$ (i.e. temperature on pathlines at $c=0.99$ ) for case $\mathrm{H}-\mathrm{D}$ is shown in Fig. B-1. The data are colored by $\mathrm{OHGrad}_{\text {norm }}=\left|\nabla \mathrm{X}_{\mathrm{OH}}\right|_{\max } /\left|\nabla \mathrm{X}_{\mathrm{OH}}\right|_{\max -1 \mathrm{D}}$ where $\left|\nabla \mathrm{X}_{\mathrm{OH}}\right|_{\max }$ is the maximum gradient of $\mathrm{X}_{\mathrm{OH}}$ on the pathlines and $\left|\nabla \mathrm{X}_{\mathrm{OH}}\right|_{\mathrm{MAX}-1 \mathrm{D}}$ is the maximum gradient of $\mathrm{X}_{\mathrm{OH}}$ from 1D laminar flame calculation. These data clearly show a general correlation between $\mathrm{OHGrad}_{\text {norm }}$ and $\mathrm{H}_{2}^{\text {con }}$ with zero or near-

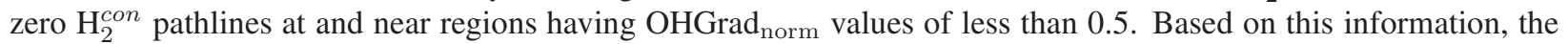
pathlines were filtered by using threshold $\mathrm{OHGrad}_{\text {norm }}$ values of $0.3,0.4$ and 0.5 . As shown by the results in Fig. B-2, the filtered curvature PDFs for the two cases are not highly sensitive to the threshold value. Consequent, we chose a 
conservative $\mathrm{OHGrad}_{\text {norm }}>0.3$ as our filter threshold. 


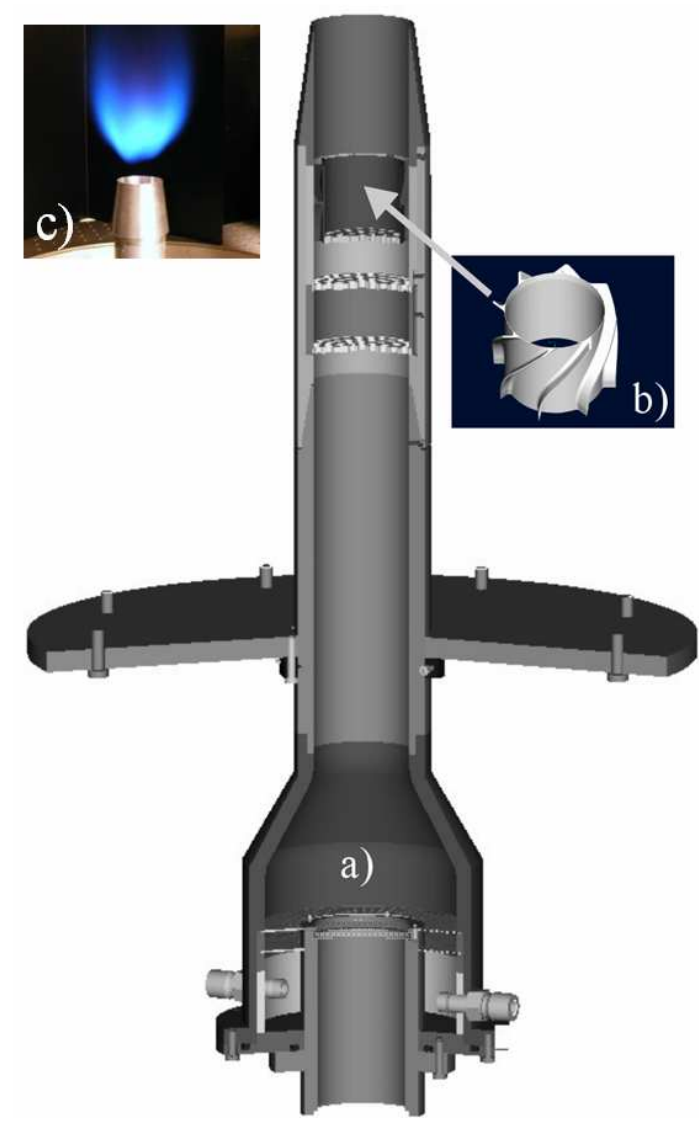

Figure 1: Schematics of the LSB device and photograph of lifted LSB flame.

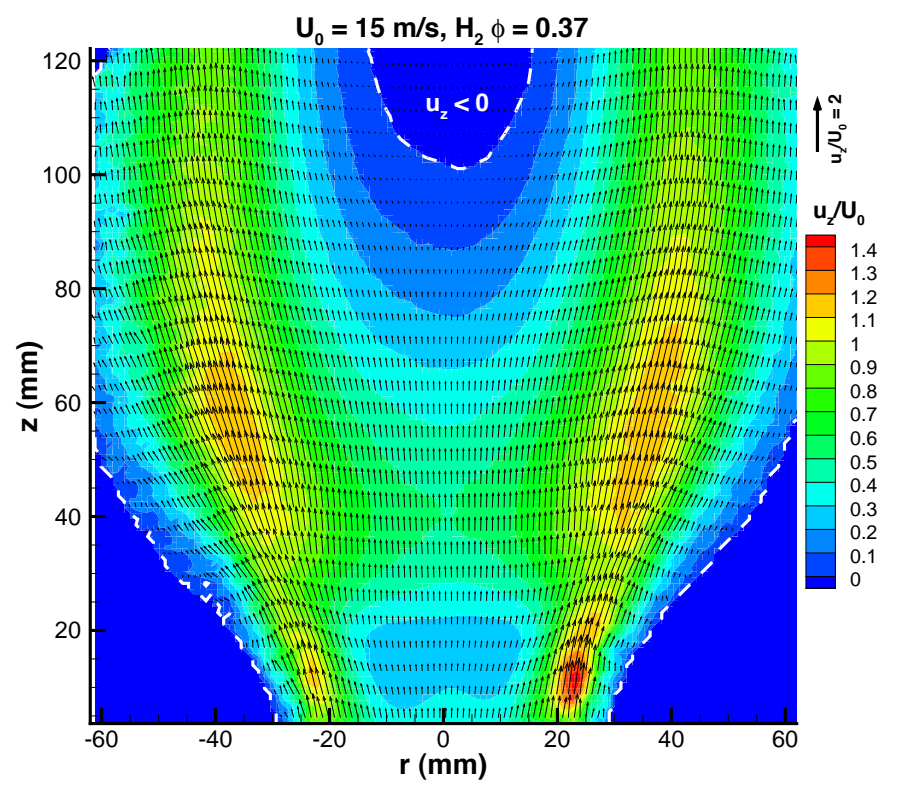

Figure 2: Measured mean velocity vectors, Case $\mathrm{H}-\mathrm{D}\left(\mathrm{H}_{2}\right.$-air fuel, $\left.\phi=0.37, U_{0}=15 \mathrm{~m} / \mathrm{s}\right)$. Contour colors indicate axial velocity magnitude. 

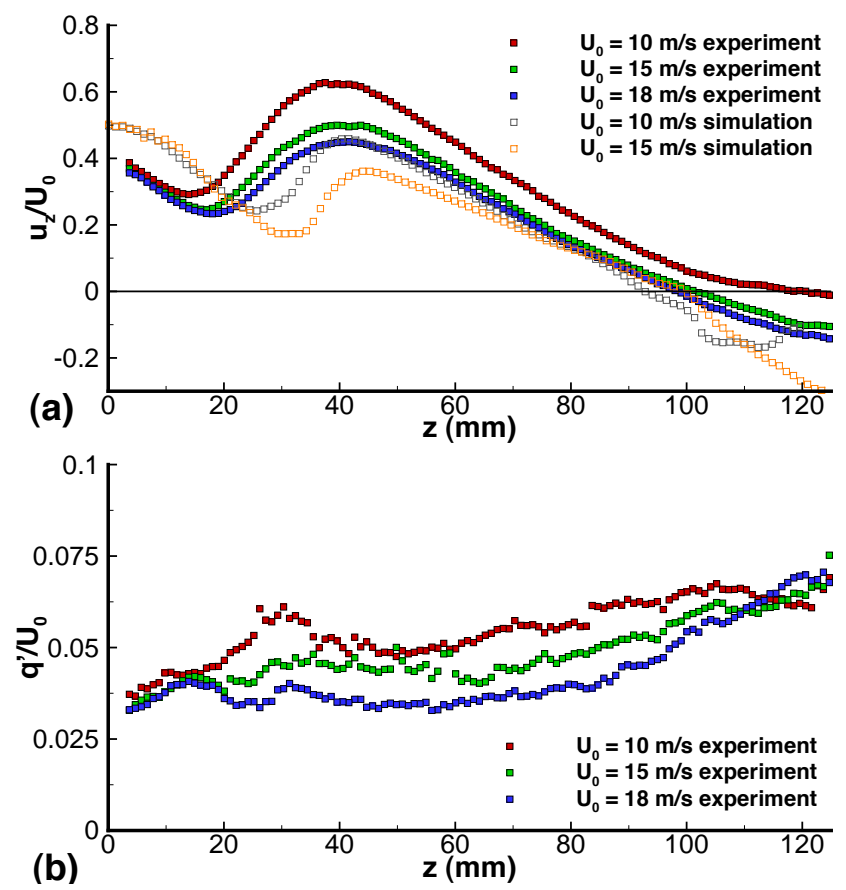

Figure 3: Mesured mean axial velocity (upper) and fluctuations (lower) along nozzle centerline $\left(\mathrm{H}_{2}\right.$-air fuel, $\phi=0.37$, $U_{0}=10,15$ and $18 \mathrm{~m} / \mathrm{s}$.)

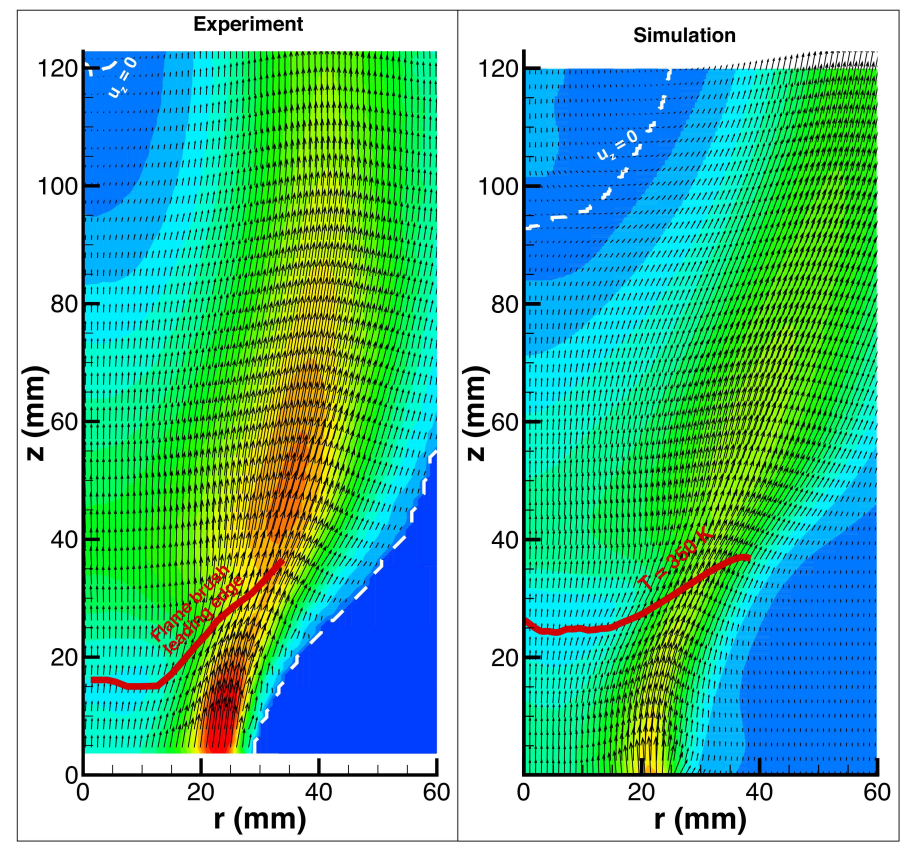

Figure 4: Measured and simulated mean velocity vectors, case $\mathrm{H}-\mathrm{C}\left(U_{0}=10 \mathrm{~m} / \mathrm{s}\right.$, legend same as Fig. 2). 


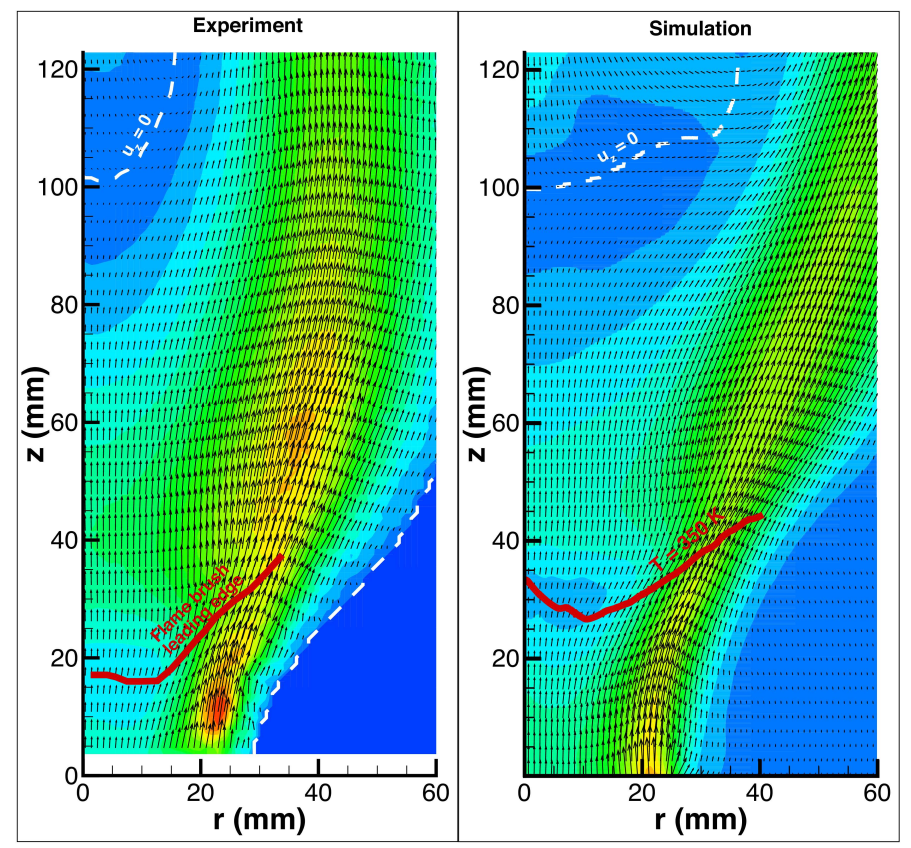

Figure 5: Measured and simulated mean velocity vectors, case H-D $\left(U_{0}=15 \mathrm{~m} / \mathrm{s}\right.$, legend same as Fig. 2).
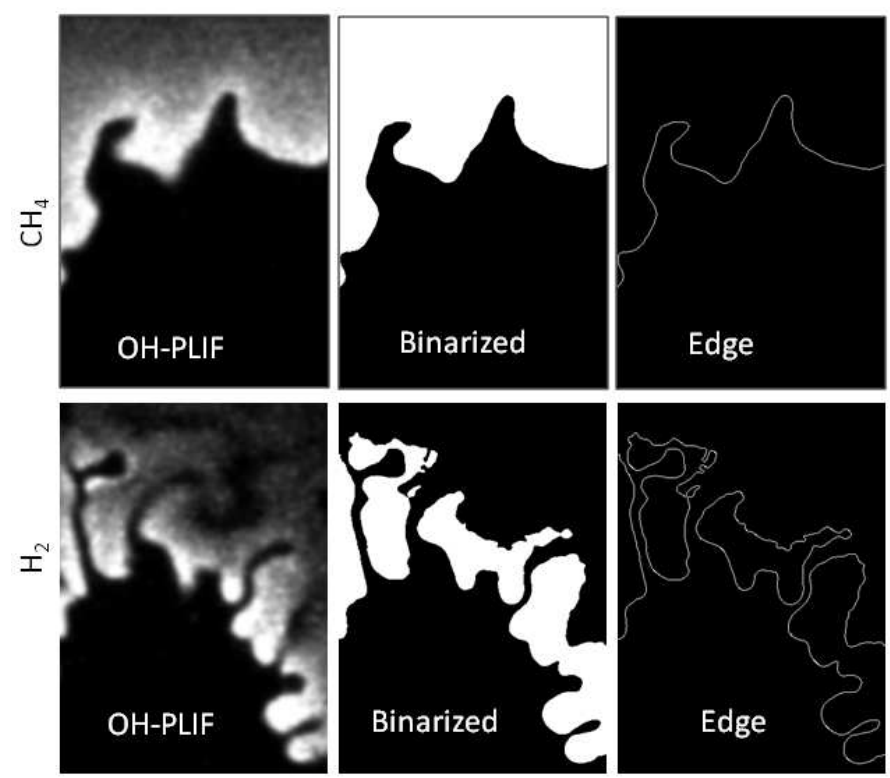

Figure 6: OH-PLIF image analysis for lean $\mathrm{CH}_{4}$ and $\mathrm{H}_{2}$ LSB flames based on signal intensity threshold. 

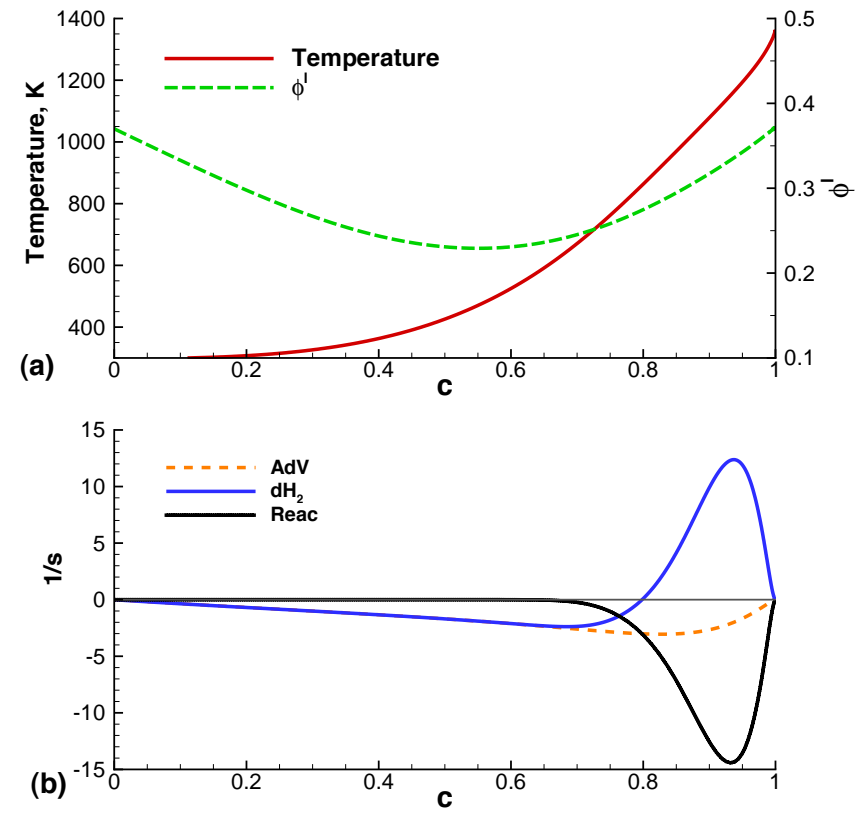

Figure 7: Reference 1D unstrained laminar flame profiles for a $\mathrm{H}_{2}$-air mixture $(\phi=0.37)$.

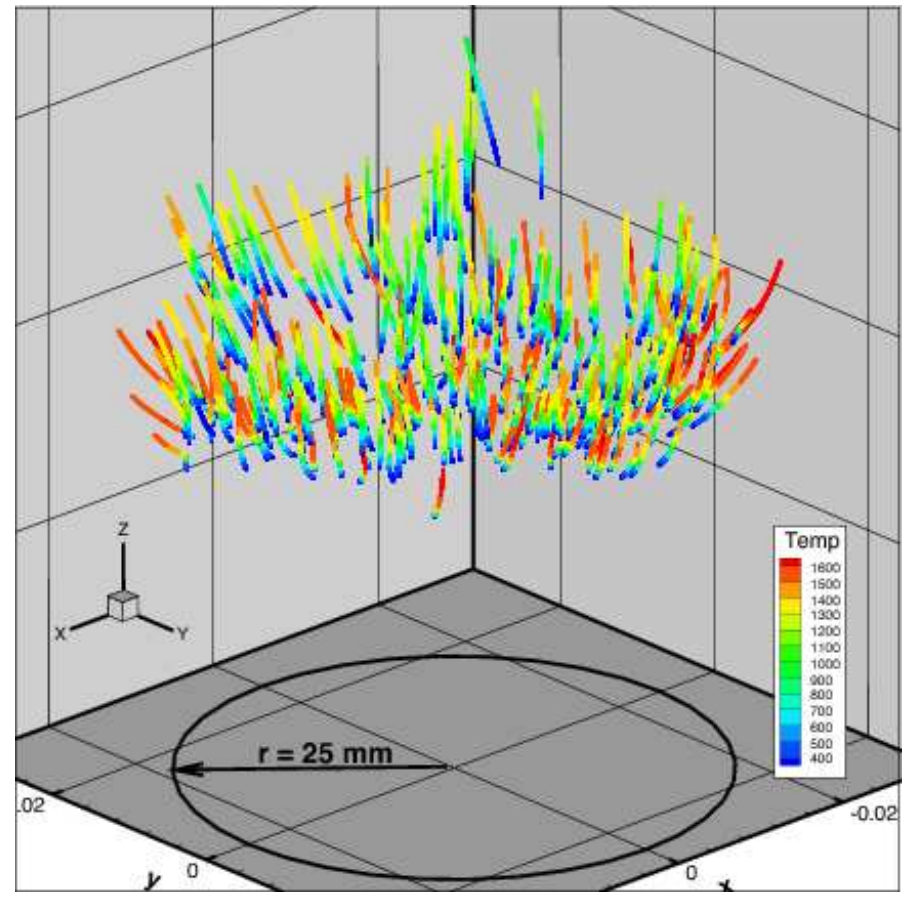

Figure 8: 300 randomly selected pathlines for simulated flame H-D $\left(U_{0}=15 \mathrm{~m} / \mathrm{s}\right)$. 

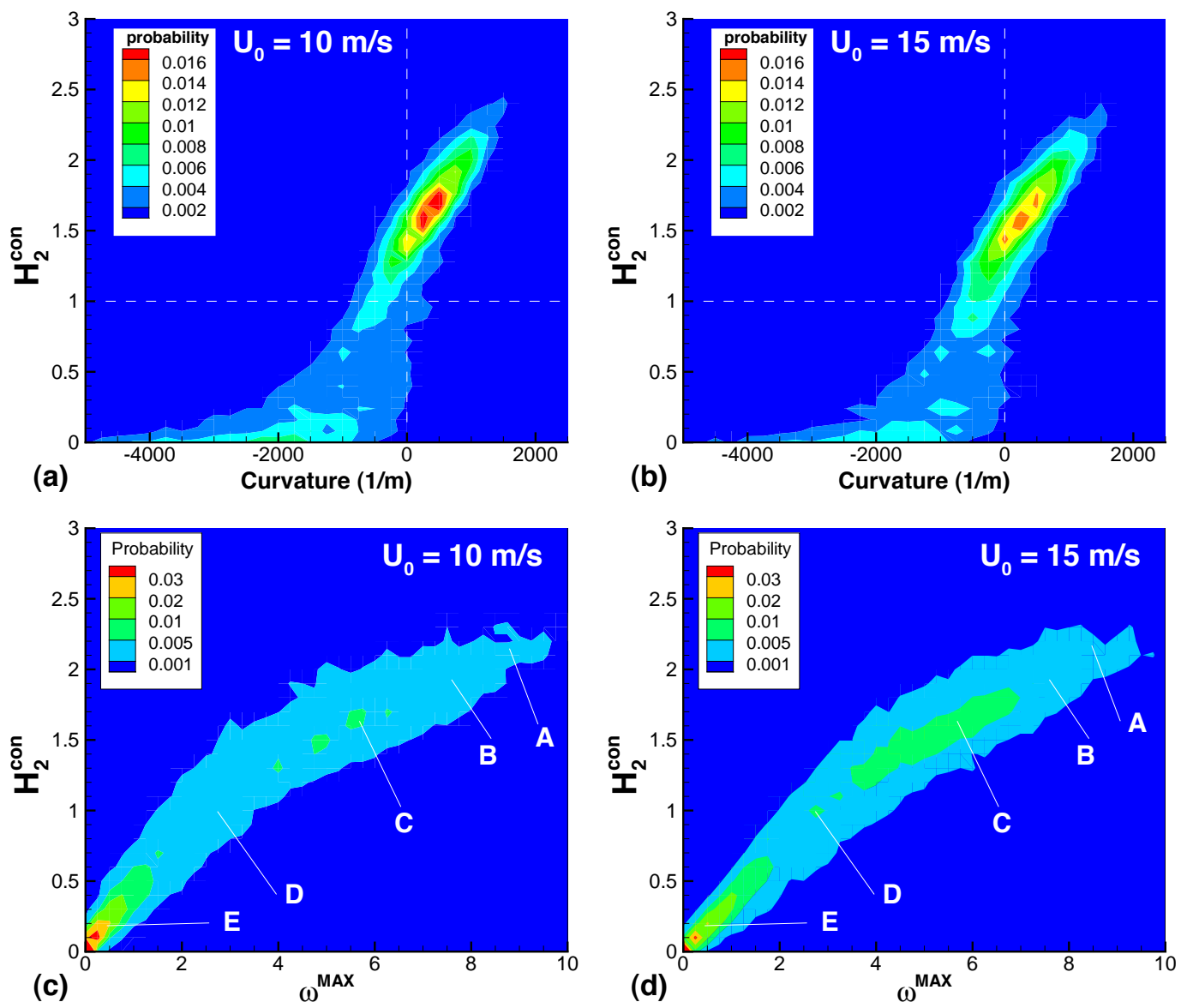

Figure 9: JPDFs for $\mathrm{H}_{2}^{\text {con }}$ and mean curvature, for (a) simulated flame $\mathrm{H}-\mathrm{C}\left(U_{0}=10 \mathrm{~m} / \mathrm{s}\right)$, (b) H-D $\left(U_{0}=15 \mathrm{~m} / \mathrm{s}\right)$. JPDFs for $\mathrm{H}_{2}^{\text {con }}$ and peak consumption rate, $\omega^{\max }$, for (c) simulated flame $\mathrm{H}-\mathrm{C}\left(U_{0}=10 \mathrm{~m} / \mathrm{s}\right)$, (d) H-D $\left(U_{0}=15 \mathrm{~m} / \mathrm{s}\right)$. 

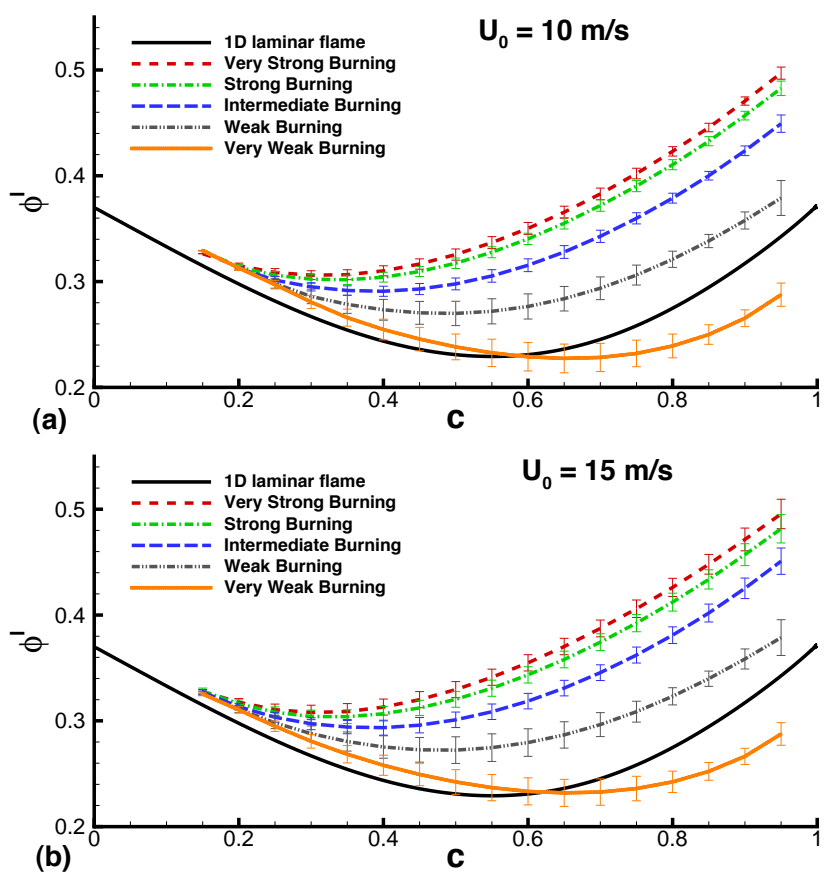

Figure 10: Local atomic stoichiometry, $\phi^{l}$ vs. $c$, in simulated flames, by pathline groups in $\mathrm{H}_{2}^{\text {con }}$ for flame $\mathrm{H}-\mathrm{C}$ (upper) and H-D (lower). 

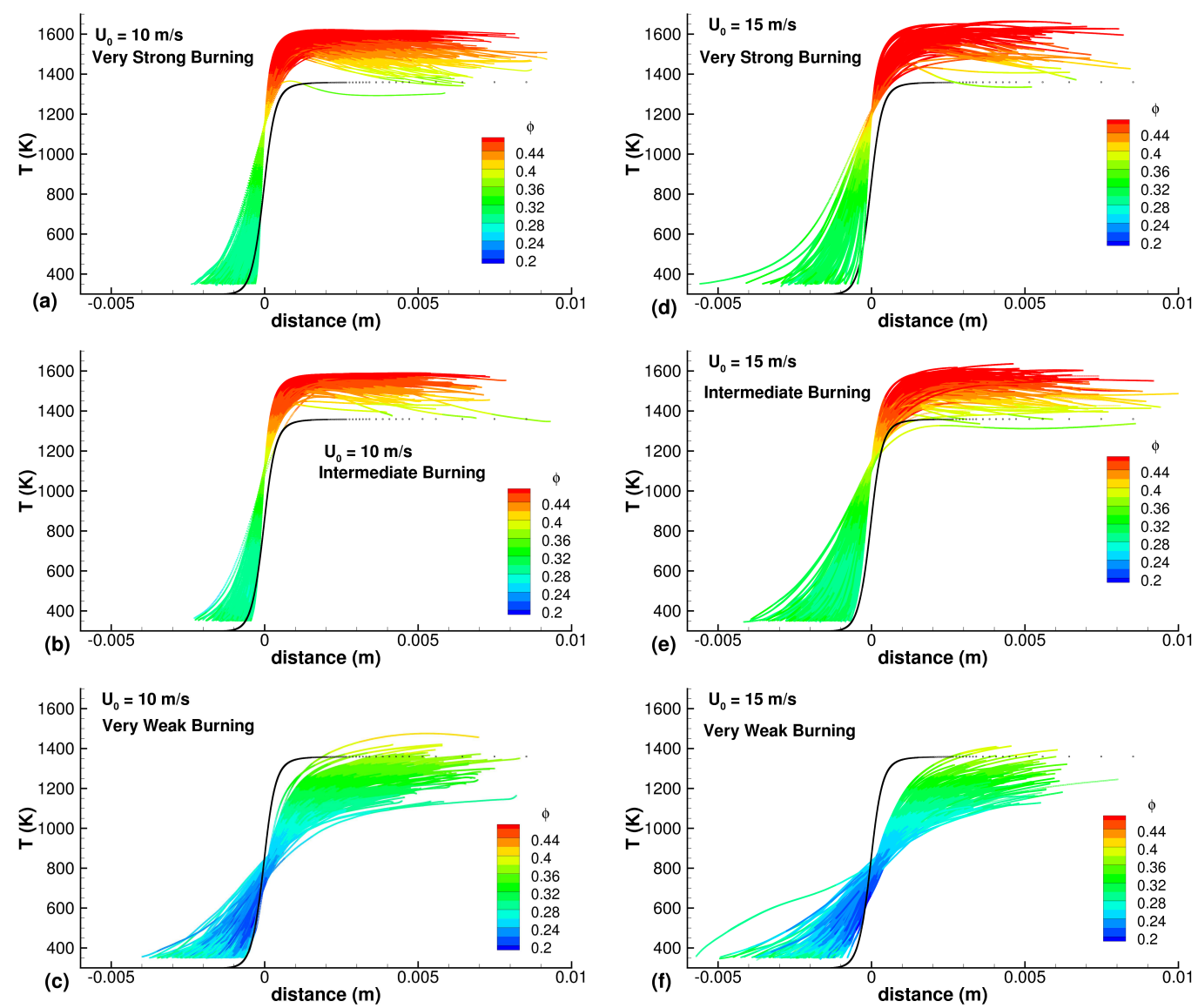

Figure 11: Temperature along pathlines in simulated flames, by pathline groups in $\mathrm{H}_{2}^{\text {con }}$ for flame $\mathrm{H}-\mathrm{C}$ (left) and $\mathrm{H}-\mathrm{D}$ (right). The unstrained profile (black line) for $\phi=0.37$ is included in each for reference. 

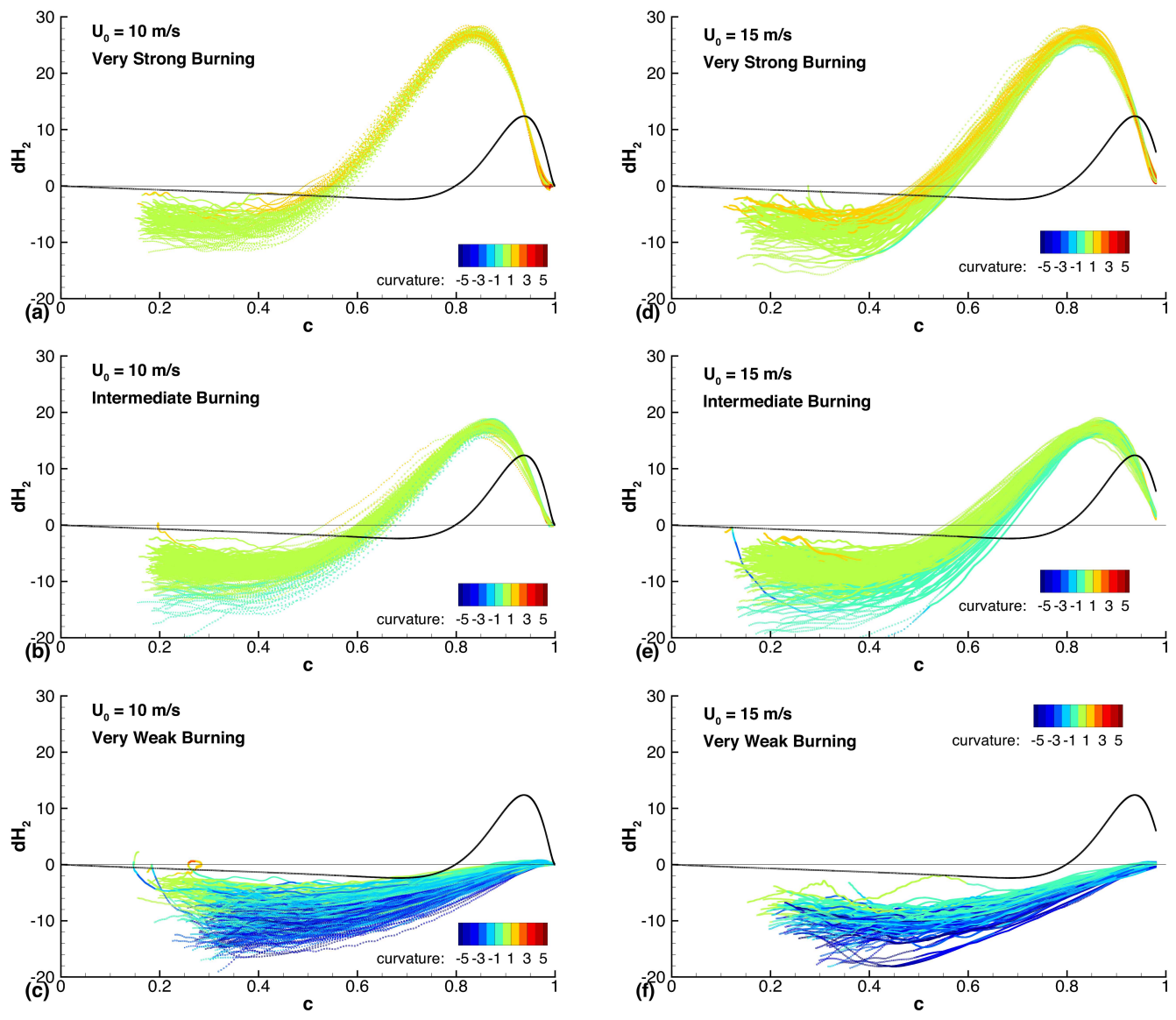

Figure 12: Diffusion flux divergence, $\mathrm{dH}_{2}$, along pathlines in simulated flames, by pathline groups in $\mathrm{H}_{2}^{\text {con }}$, colored by contours in mean curvature for flame H-C (left) and H-D (right). The unstrained profile (black line) for $\phi=0.37$ is included in each for reference. 

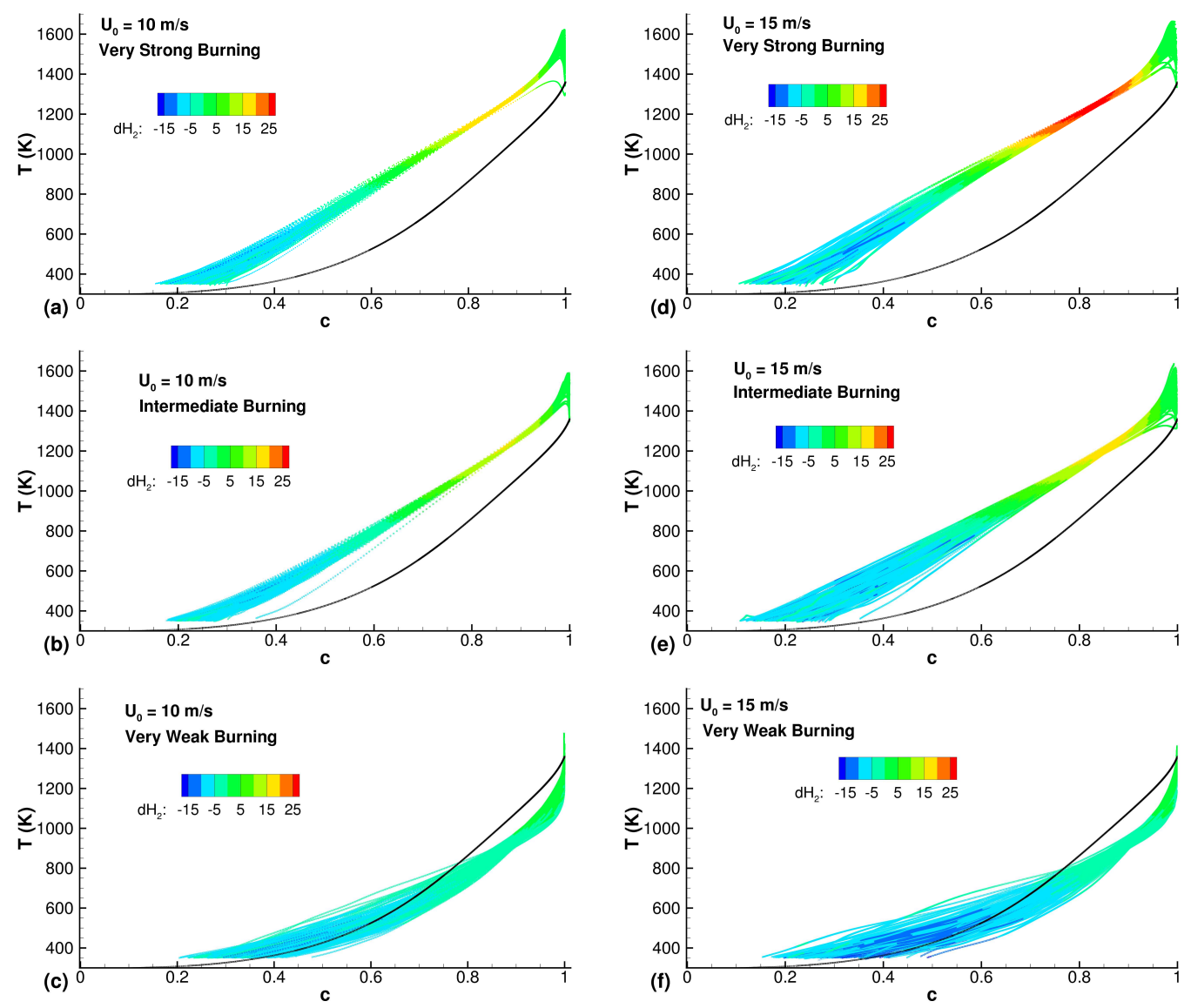

Figure 13: Temperature verses $c$ in simulated flames, by pathline groups in $\mathrm{H}_{2}^{\text {con }}$ for flame $\mathrm{H}-\mathrm{C}$ (left) and $\mathrm{H}-\mathrm{D}$ (right). The unstrained profile (black line) for $\phi=0.37$ is included in each for reference. 

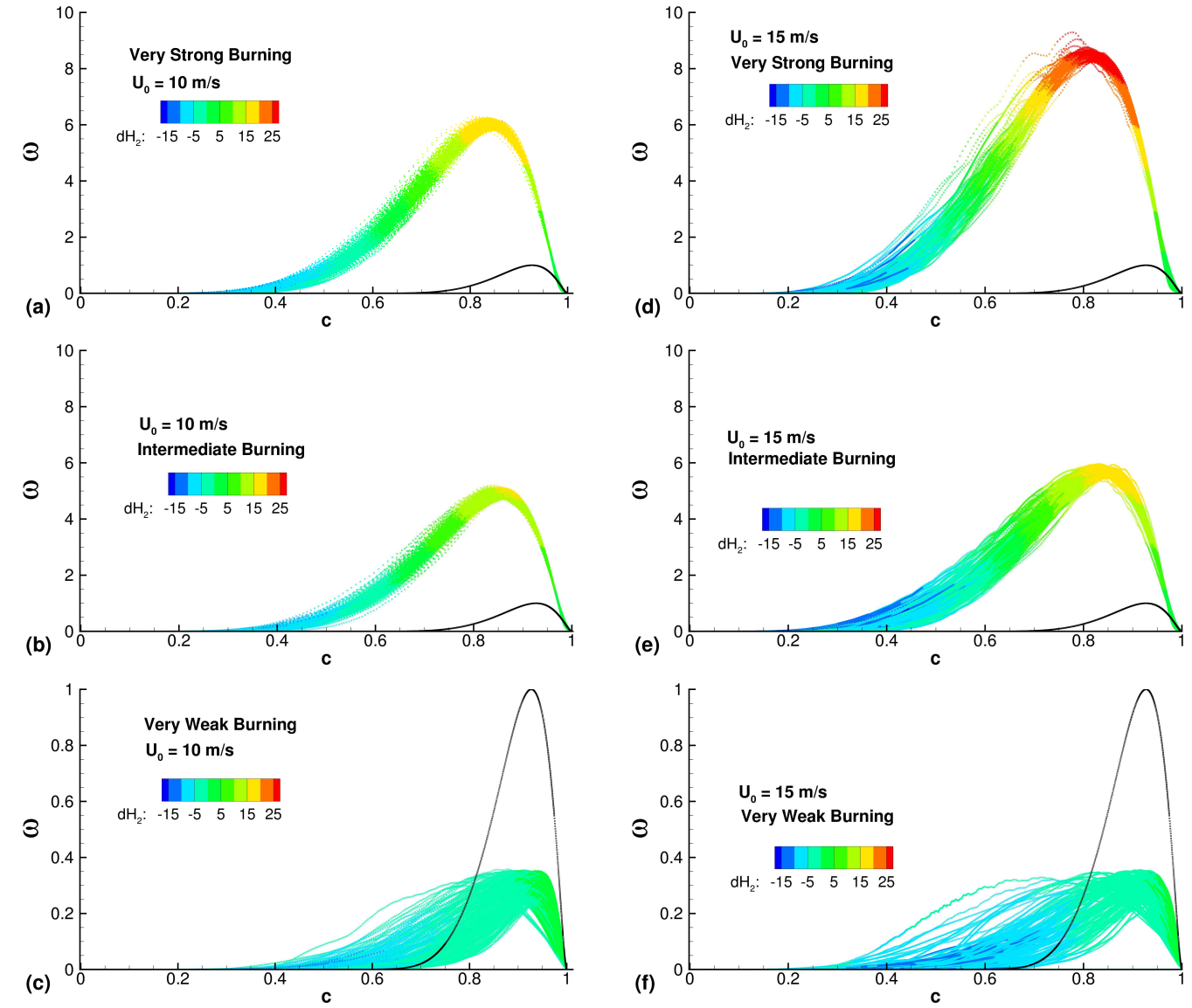

Figure 14: Locally normalized consumption rate, $\omega$, in simulated flames, by pathline groups in $\mathrm{H}_{2}^{\text {con }}$ for flame $\mathrm{H}-\mathrm{C}$ (left) and H-D (right). The unstrained profile (black line) for $\phi=0.37$ is included in each for reference. 

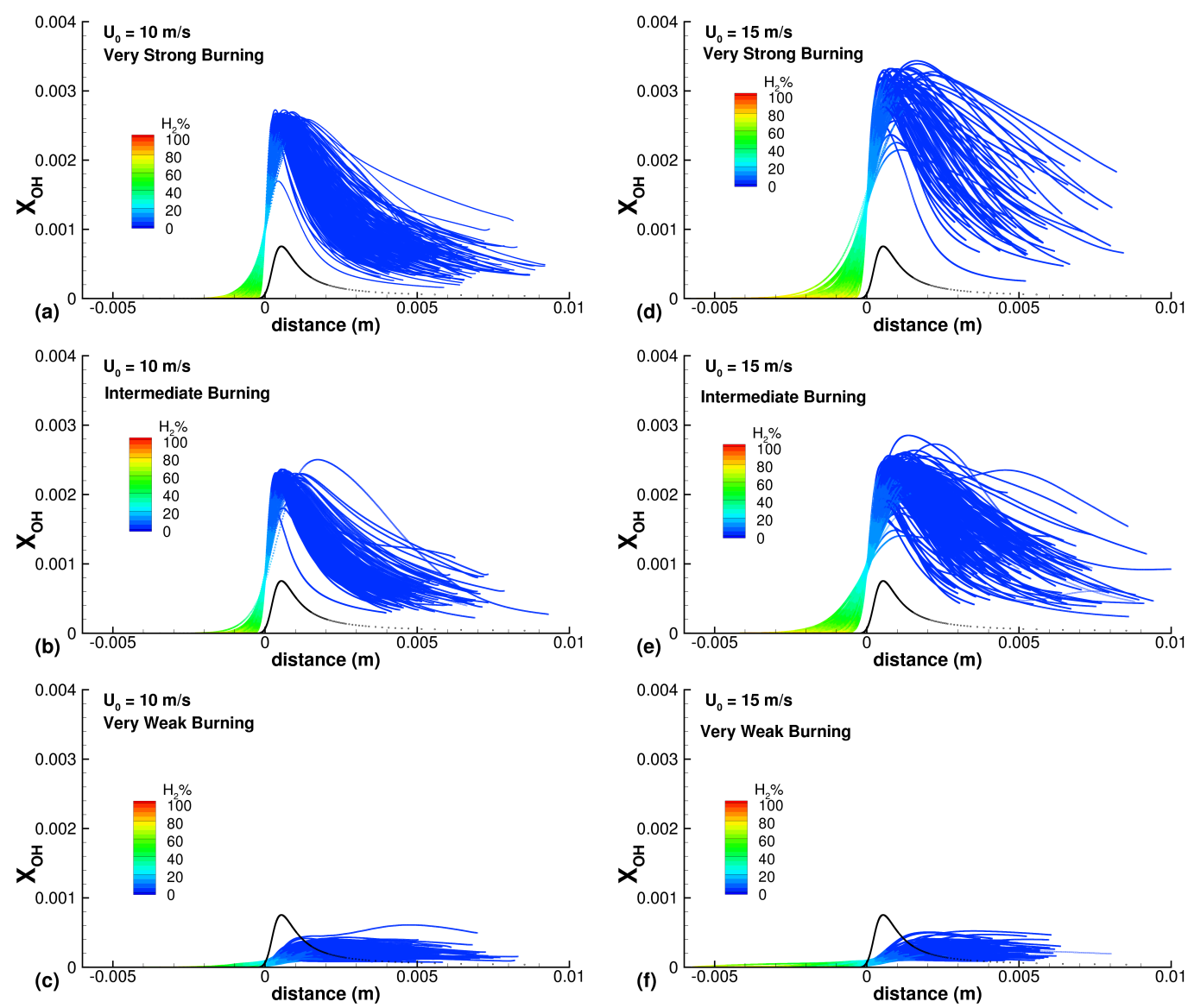

Figure 15: $\mathrm{OH}$ profiles along pathlines in simulated flames, by pathline groups in $\mathrm{H}_{2}^{\text {con }}$, colored by fraction of fuel remaining on each line, for flame H-C (left) and H-D (right). The unstrained profile (black line) for $\phi=0.37$ is included in each for reference.
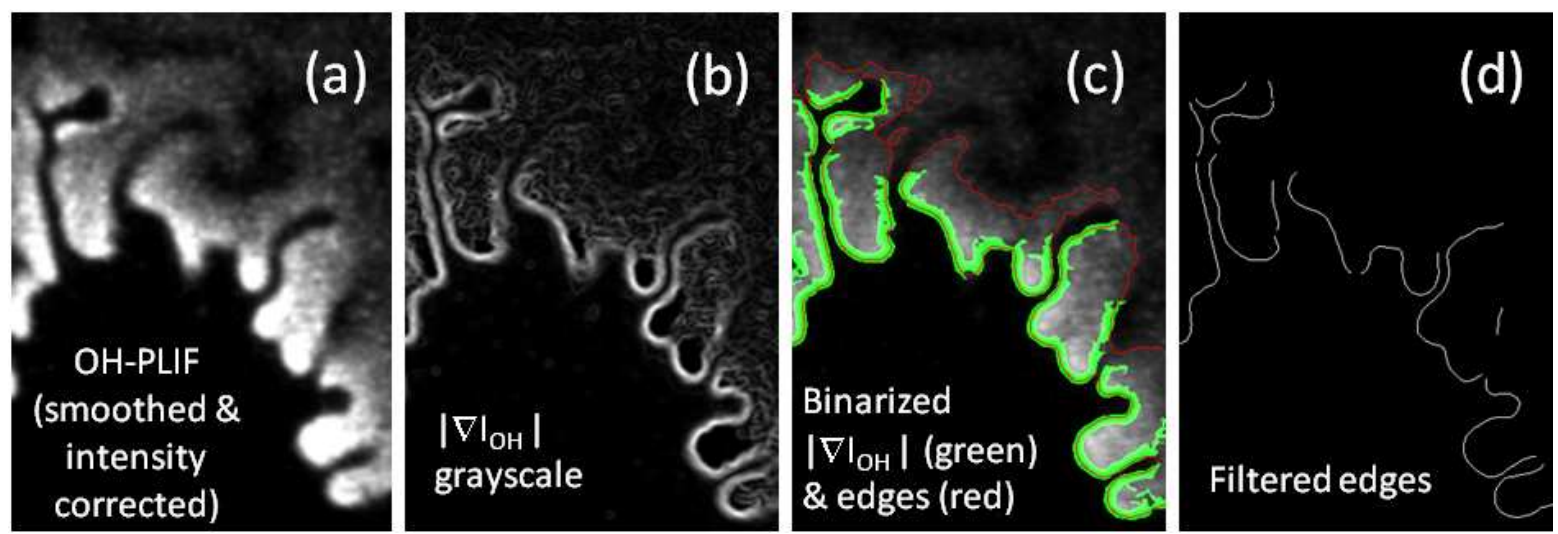

Figure 16: OH-PLIF analysis steps: (a) Normalized and spatial filtered OH-PLIF, (b) $\left|\nabla I_{\mathrm{OH}}\right|$ grayscale (OH-PLIF was smoothed by a $5 \times 5$ pixel Gauassian filter before applying the gradient operation.), (c) $\left|\nabla I_{\mathrm{OH}}\right| 25 \%$ peak threshhold (green-overlay) and edges of high OH-PLIF intensity (red), (d) product of images (b) and (c). 

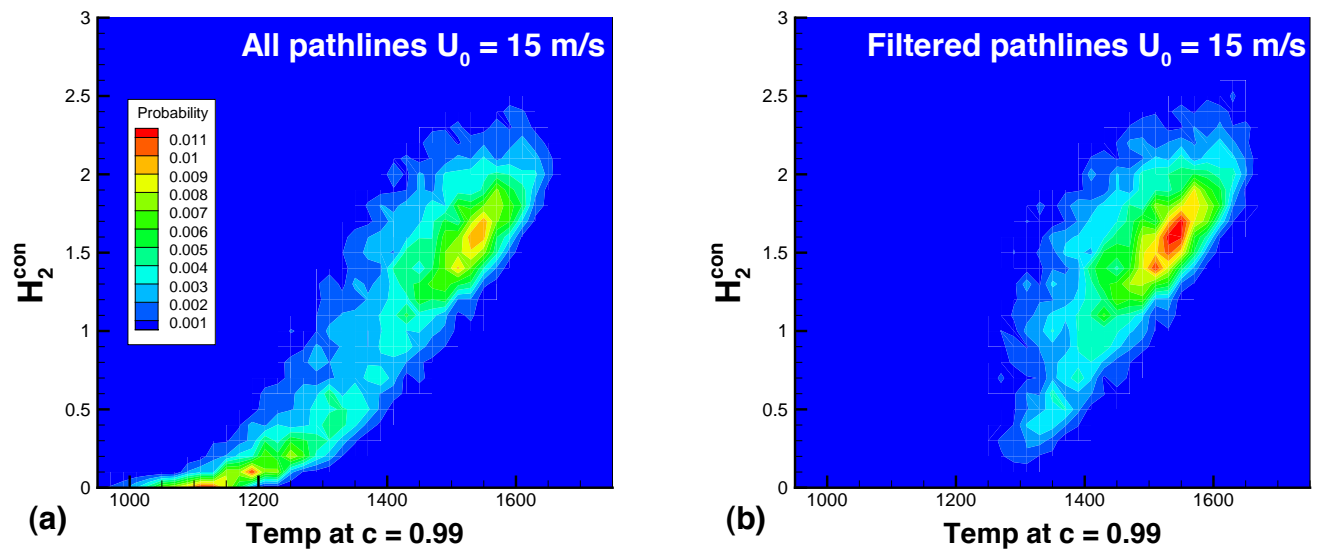

Figure 17: JPDF of $\omega_{\max }$ and $T_{c=0.99}$ before (left) and after (right) filtering.
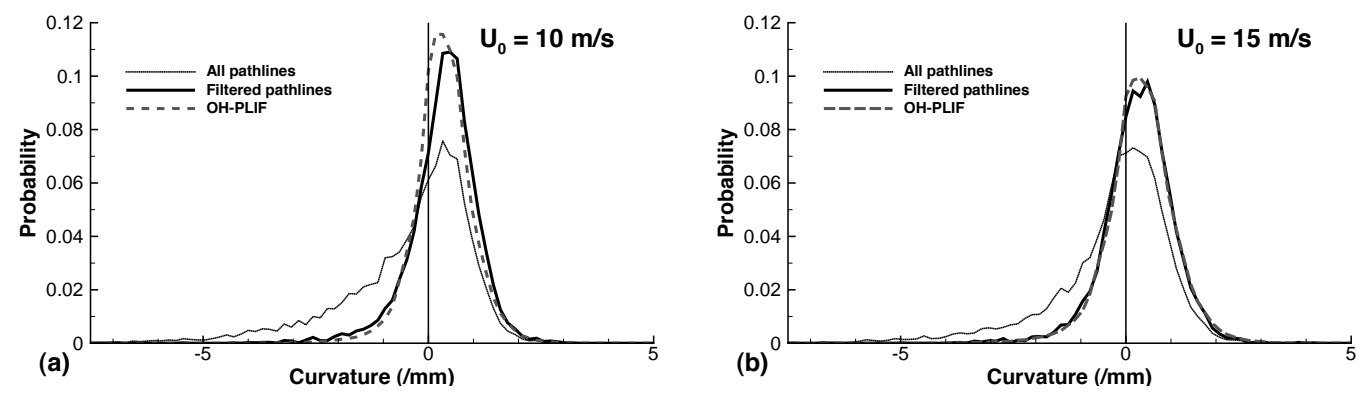

Figure 18: Comparison of two-dimensional curvature, $\kappa_{2 D}$, filtered, unfiltered (simulated) and measured OH-PLIF data for the two cases, $10 \mathrm{~m} / \mathrm{s}$ (left), and $15 \mathrm{~m} / \mathrm{s}$ (right). 


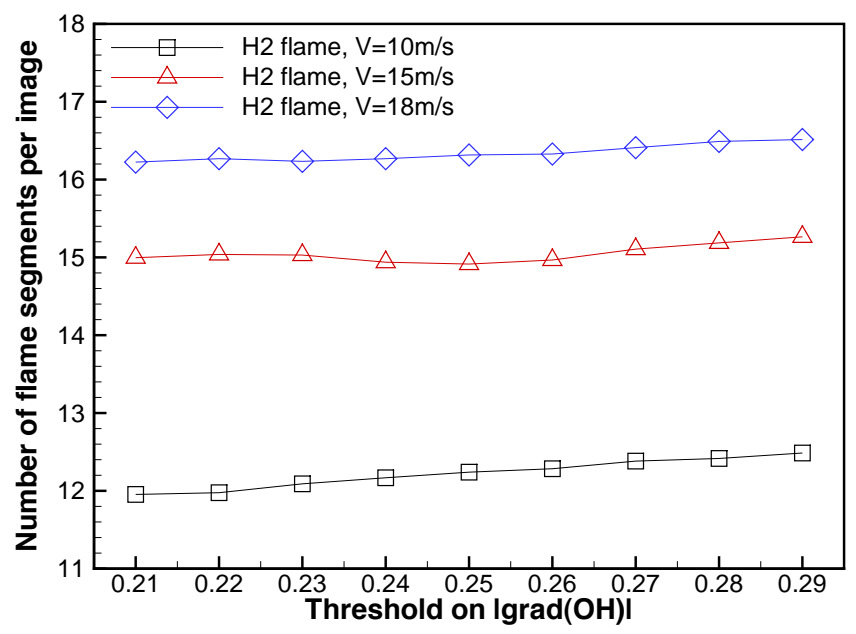

Figure A-1: Number of flame segments per image for different threshold values of $\left|\nabla \mathrm{I}_{\mathrm{OH}}\right|$.

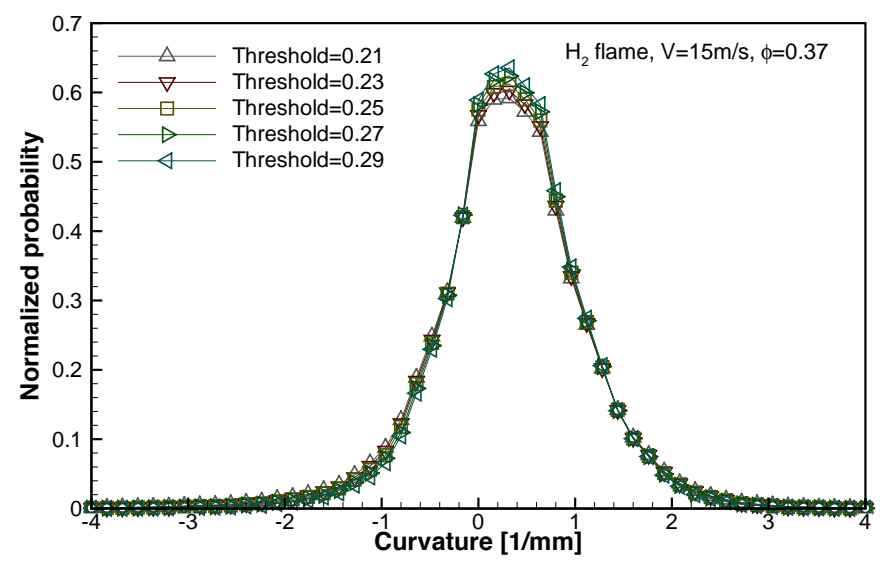

Figure A-2: Two-dimensional curvature pdfs for different threshold values of $\left|\nabla \mathrm{I}_{\mathrm{OH}}\right|$. 